\title{
Bone Morphogenetic Protein Inhibition Promotes Neurological Recovery after Intraventricular Hemorrhage
}

\author{
Krishna Dummula, ${ }^{1,8 \star}$ Govindaiah Vinukonda, ${ }^{1,2 \star}$ Philip Chu, ${ }^{3}$ Yiping Xing, ${ }^{1}$ Furong Hu, ${ }^{1}$ Sabrina Mailk, ${ }^{1}$ \\ Anna Csiszar, ${ }^{4}$ Caroline Chua, ${ }^{1}$ Peter Mouton, ${ }^{5}$ Robert J. Kayton, ${ }^{6}$ Joshua C. Brumberg, ${ }^{3}$ Rashmi Bansal, ${ }^{7}$ and \\ Praveen Ballabh ${ }^{1,2}$ \\ Departments of ${ }^{1}$ Pediatrics and ${ }^{2}$ Anatomy and Cell Biology, New York Medical College-Westchester Medical Center, Valhalla, New York 10595, \\ ${ }^{3}$ Department of Psychology, Queens College, City University of New York, Flushing, New York 11367, ${ }^{4}$ Reynolds Oklahoma Center on Aging, Department of \\ Geriatric Medicine, University of Oklahoma Health Science Center, Oklahoma City, Oklahoma 73104, ${ }^{5}$ Department of Pathology and Cell Biology, University \\ of South Florida School of Medicine, Tampa, Florida 33612, ${ }^{\circ}$ Department of Physiology and Pharmacology, Oregon Health \& Science University, Portland, \\ Oregon 97239, ${ }^{7}$ Department of Neuroscience, University of Connecticut Medical School, Farmington, Connecticut 06030, and ${ }^{8}$ Department of Pediatrics, \\ University of Kansas, School of Medicine, Kansas City, Kansas 66160
}

Intraventricular hemorrhage (IVH) results in neural cell death and white matter injury in premature infants. No therapeutic strategy is currently available against this disorder. Bone morphogenetic protein (BMP) signaling suppresses oligodendrocyte development through basic-helix-loop-helix (bHLH) transcription factors and promotes astrocytosis. Therefore, we hypothesized that IVH in premature newborns initiates degeneration and maturation arrest of oligodendrocyte lineage and that BMP inhibition alleviates hypomyelination, gliosis, and motor impairment in the survivors of IVH. To test the hypotheses, a rabbit model of IVH was used in which premature rabbit pups (E29) are treated with intraperitoneal glycerol at $2 \mathrm{~h}$ of age to induce IVH; and the pups with IVH exhibit hypomyelination and gliosis at 2 weeks of postnatal age. Maturation of oligodendrocyte lineage was evaluated by specific markers, and the expression of bHLH transcription factors was assessed. BMP levels were measured in both premature rabbit pups and autopsy materials from premature infants. Recombinant human noggin was used to suppress BMP action; and neurobehavioral performance, myelination and gliosis were assessed in noggin-treated pups compared with untreated controls. We found that IVH resulted in apoptosis and reduced proliferation of oligodendrocyte progenitors, as well as arrested maturation of preoligodendrocytes in rabbits. BMP4 levels were significantly elevated in both rabbit pups and human premature infants with IVH compared with controls. Importantly, BMP inhibition by recombinant human noggin restored the levels of phospho-Smad1/5/8, Olig2 transcription factor, oligodendrocyte maturation, myelination, astrocyte morphology, and motor function in premature pups with IVH. Hence, BMP inhibition might enhance neurological recovery in premature infants with IVH.

\section{Introduction}

Approximately 12,000 premature infants develop intraventricular hemorrhage (IVH) every year in the USA alone (Heuchan et al., 2002). The hemorrhage typically originates in the germinal matrix and often progresses to IVH with the rupture of the ventricular ependyma. The germinal matrix, located on the head of

Received Jan. 1, 2011; revised March 10, 2011; accepted May 9, 2011.

Author contributions: J.C.B., R.B., and P.B. designed research; K.D., G.V.,P.C., Y.X., F.H., S.M., A.C., C.C., R.J.K., and

P.B. performed research; K.D., P.R.M., and P.B. analyzed data; G.V., J.C.B., R.B., and P.B. wrote the paper.

This work was supported by NIH/NINDS Grants R01 NS071263 (P.B.) and NS 058758 (J.C.B.), American Heart Association Grant-in-Aid 09GRNT2310147 (P.B.), and NIH Grant NS38878 (R.B.). We thank Joanne Abrahams from New York Medical College for technical assistance with images and Elizabeth Flannery from the Department of Physiology and Pharmacology, Oregon Health \& Science University, for technical assistance with electron microscopy. We acknowledge the electron microscopy performed by the Neuroscience Imaging Center's Electron Microscopy Core at Oregon Health \& Science University.

*K.D. and G.V. contributed equally to the manuscript.

The authors declare no competing financial interests.

Correspondence should be addressed to Dr. Praveen Ballabh, Regional Neonatal Center, Maria Fareri Children's Hospital at Westchester Medical Center, Valhalla, NY 10595. E-mail: Pballabh@msn.com.

DOI:10.1523/JNEUROSCI.0013-11.2011

Copyright $\odot 2011$ the authors $\quad 0270-6474 / 11 / 3112068-15 \$ 15.00 / 0$ caudate nucleus beneath the ventricular ependyma, is a vascularized collection of neuronal-glial precursor cells and is selectively vulnerable to hemorrhage. IVH is associated with white matter injury, manifesting as cerebral palsy and cognitive deficits in the survivors (Armstrong et al., 1987; Bassan et al., 2007). No therapeutic or preventive strategy is currently available for the white matter injury in premature infants with IVH. Hence, we asked what the mechanism of white matter injury in IVH was and how this could be prevented.

During human pregnancy, 23-35 weeks of gestation is the window when oligodendrocyte $(\mathrm{OL})$ lineage progresses through distinct phenotypic stages-OL progenitors, pre-OL, immature and then mature OL (Back et al., 2001). OL progenitors are identified by reactivity to PDGFR $\alpha$ or NG2 antibody, pre-OLs are reactive to $\mathrm{O} 4$ monoclonal antibody; and the immature OLs express galactocerebroside (O1 antigen) and $2^{\prime}, 3^{\prime}$-cyclic nucleotide $3^{\prime}$-phosphodiesterase (CNPase). The mature OLs synthesize the major myelin proteins - myelin basic protein and proteolipid protein (Pfeiffer et al., 1993). Pre-OLs are vulnerable to oxidative stress and to hypoxic-ischemic insults, whereas immature OLs 
are relatively resistant to these events (Back et al., 2005). Acute hypoxia-ischemia in neonatal rats results in degeneration and maturation arrest of pre-OLs, rendering hypomyelination in the hypoxic-ischemic white matter (Segovia et al., 2008). However, the distinctive changes in the transcriptional network that might cause developmental arrest of OLs in neonatal models of brain injury remains unknown.

The maturation of OLs is regulated by basic helix-loop-helix (bHLH) transcription factors, including Olig1, Olig2, Id2, Id4, and Sox10 (Nicolay et al., 2007). Loss- and gain-of-function experiments have shown that these factors regulate OL differentiation (Lu et al., 2002; Xin et al., 2005). BMP signaling pathways are important regulators of the bHLH transcriptional factors and oligodendrogenesis (Hall and Miller, 2004; See et al., 2007). Although BMP is necessary for OL lineage maturation, BMP overexpression inhibits OL lineage progression by suppressing Olig1 and Olig2 transcription factors (Nicolay et al., 2007). BMP promotes astrocytosis and inhibits OL lineage commitment by diverting oligodendroglial precursors into astrocytic progenitors (Gomes et al., 2003). On this basis, we hypothesized that IVH in premature newborns would result in OL degeneration and distinctive disturbances in the levels of bHLH transcriptional factors, thereby arresting maturation of pre-OLs. We also postulated that BMP might be elevated in IVH, and that BMP inhibition would restore the transcription factors, maturation of OLs, myelination, astrocyte morphology, and neurological function in the survivors of IVH. To test these hypotheses, we used our animal model, in which rabbit pups with glycerol-induced IVH exhibit hypomyelination and gliosis at 2 weeks of postnatal age (Chua et al., 2009).

Here we show that IVH resulted in apoptosis, reduced proliferation, and arrested maturation of pre-OLs. BMP4 levels were elevated in newborns with IVH than controls; and BMP inhibition restored myelination and neurological recovery in pups with IVH.

\section{Materials and Methods}

Animal experiments. The Institutional Animal Care and Use Committee of New York Medical College approved the use of animals for this study. We obtained timed-pregnant New Zealand rabbits from Charles River Laboratories. C-section was performed to deliver the pups prematurely at E29 (full-term $=32 \mathrm{~d}$ ). We kept them in an infant incubator prewarmed to a temperature of $35^{\circ} \mathrm{C}$. The pups were gavage-fed $1 \mathrm{ml}$ of rabbit milk at $4 \mathrm{~h}$ of age and then $\sim 2 \mathrm{ml}$ every $12 \mathrm{~h}(100 \mathrm{ml} / \mathrm{kg} / \mathrm{d})$ for the first $2 \mathrm{~d}$. Subsequently, they were fed puppy formula (Esbilac), and feeds were advanced to $125,150,200,250$, and $280 \mathrm{ml} / \mathrm{kg}$ at postnatal days 3,5 , 7,10 , and 14 , respectively. The rabbit pups of either sex were administered $50 \%$ glycerol $(6.5 \mathrm{~g} / \mathrm{kg})$ intraperitoneally at $2 \mathrm{~h}$ of age to induce IVH. Head ultrasound was performed at $6 \mathrm{~h}$ of age to evaluate the presence and severity of IVH using an Acuson Sequoia C256 (Siemens) ultrasound machine. We classified IVH as (1) mild, not gross but microscopic hemorrhage detected in H\&E-stained brain sections; (2) moderate, gross hemorrhage into lateral ventricles (two separate lateral ventricles visualized); or (3) severe, gross IVH leading to fusion of lateral ventricles into a common chamber (Fig. 1 A) (Vinukonda et al., 2010). As microscopic IVH cannot be diagnosed by head ultrasound, an absence of IVH in glycerol-treated pups indicated that the kit had either microscopic or no IVH.

Noggin treatment. To inhibit BMP action, we treated rabbit pups with human recombinant noggin (Invitrogen), starting at $24 \mathrm{~h}$ of age. The severity of IVH was similar between the comparison groups-vehicletreated pups with IVH and noggin-treated pups with IVH. Briefly, pups were restrained after being anesthetized with ketamine $(35 \mathrm{mg} / \mathrm{kg}, \mathrm{i} . \mathrm{m}$.) and xylazine $(5 \mathrm{mg} / \mathrm{kg}$, i.m.). Intracerebroventricular (ICV) cannula (catalog \#0004760, Alzet, Durect ) was implanted in cerebral ventricle using the following coordinates from bregma: $1 \mathrm{~mm}$ anterior, $4 \mathrm{~mm}$ lateral, and $3 \mathrm{~mm}$ deep. The cannula was connected to an Alzet miniosmotic pump (model 1003D $1 \mu \mathrm{l} / \mathrm{hx} 3 \mathrm{~d}$; model $20011 \mu \mathrm{l} / \mathrm{hx} 7 \mathrm{~d}$ ), and the pump was implanted between shoulder blades. Those pups, who were killed at the end of day 3, received noggin infusion for $3 \mathrm{~d}$ (800 $\mathrm{ng}$ of noggin in $100 \mu \mathrm{l}$ of saline); and pups, who were killed at day 7 or 14 , received noggin infusion for $7 \mathrm{~d}$ (1600 ng of noggin in $200 \mu \mathrm{l}$ of saline).

Human subjects. The Institutional Review Board of New York Medical College (Valhalla, NY) approved the use of human autopsy materials for this study. The study materials included forebrain tissue samples taken from premature infants with and without IVH of 23-26 weeks of gestational age and 3-5 d of postnatal age. Samples were obtained $<24 \mathrm{~h}$ postmortem. We excluded premature infants with meningitis, hypoxic-ischemic encephalopathy, culture proven sepsis, major congenital anomalies, and chromosomal defects. We included six infants in each group-IVH and no IVH. The wall of the cerebral hemisphere in premature infants consists of ventricular zone (VZ), subventricular zone (SVZ), intermediate zone, cortical plate, and marginal zone as described by the Boulder Committee (Bystron et al., 2008). In this study, we described intermediate-zone embryonic white matter synonymously with white matter and cortex for the cortical plate. Brain samples were processed as described previously (Vinukonda et al., 2010).

Rabbit tissue collection and processing. We processed the tissues as previously described (Ballabh et al., 2007). The brain slices were immersionfixed in $4 \%$ paraformaldehyde in PBS $(0.01 \mathrm{M}, \mathrm{pH} 7.4)$ for $18 \mathrm{~h}$ and then were cryoprotected by immersing into $20 \%$ sucrose in $0.01 \mathrm{M}$ PBS buffer for $24 \mathrm{~h}$ followed by $30 \%$ sucrose for the next $24 \mathrm{~h}$. Tissues were frozen into optimum cutting temperature compound (Sakura). Frozen coronal blocks were cut on a cryostat into $12 \mu \mathrm{m}$ sections.

Human tissue collection and processing. We processed the tissues as described previously (Ballabh et al., 2007). Approximately 2- to 3-mmthick coronal slices were taken at the level of thalamostriate groove from the frontal lobe. The coronal blocks from the frontal lobe included the cortex, white matter, and germinal matrix. The samples were fixed in $4 \%$ paraformaldehyde in PBS overnight and were then cryoprotected by immersing into a $20 \%$ sucrose solution in PBS. The tissues were frozen after embedding them into optimum cutting temperature compound (Sakura). Frozen coronal blocks were cut into $15 \mu \mathrm{m}$ sections. For Western blot analyses, pieces of tissues were directly harvested from the cortex, white matter, and germinal matrix and were frozen immediately on dry ice.

Immunohistochemistry. Immunostaining was performed as described previously (Ballabh et al., 2007). The primary antibodies used in experiments included goat polyclonal BMP2 (catalog \#sc-6895, Santa Cruz Biotechnology), goat polyclonal BMP4 (catalog \#sc-6896, Santa Cruz Biotechnology), rabbit monoclonal Ki67 (catalog \#275R-14, Cell Marque), goat polyclonal Olig2 (catalog \#AF-2418, R\&D Systems), rabbit polyclonal Olig1 (catalog \#sc-48787, Santa Cruz Biotechnology), mouse monoclonal GFAP (catalog \#G6171; Sigma-Aldrich), rat monoclonal myelin basic protein (catalog \#AB7439, Abcam), mouse monoclonal caspase-3 (clone 3CSP01, catalog \#MS1121-P; Thermo Scientific), goat polyclonal PDGFR $\alpha$ (catalog \#AF307 NA, R\&D Systems), mouse monoclonal NG2 (catalog \#5384, Millipore), mouse monoclonal Adenomatous Polyposis Coli, CC1 clone (APC) antibody (catalog \#OP80, EMD Chemicals). Fluorescent double labeling with $\mathrm{O} 4$ and $\mathrm{O} 1$ antibody (courtesy of Dr. Rashmi Bansal, University of Connecticut, Farmington, CT) used biotinylated $\mathrm{O} 4$ antibody. The secondary antibodies used were $\mathrm{Cy} 3$ conjugate donkey anti-mouse, $\mathrm{Cy} 3$ conjugate donkey anti-goat and FITC conjugate donkey anti-rat (Jackson Immunoresearch). Briefly, we hydrated the fixed sections in $0.01 \mathrm{M}$ PBS and incubated with the primary antibodies diluted in PBS at $4^{\circ} \mathrm{C}$ overnight. After washing in PBS, the sections were incubated with secondary antibody diluted in $1 \%$ normal goat serum in PBS at room temperature for $60 \mathrm{~min}$. Finally, after washes in PBS, sections were mounted with Slow Fade Light Antifade reagent (Invitrogen) and were visualized under a confocal microscope (Nikon Instruments). Stereology was performed using a fluorescent microscope (Axioskop 2 plus; Carl Zeiss) with motorized specimen stage for automated sampling (Applied Scientific Instrumentation), CCD color video camera (Microfire; Optronics), and stereology software (Stereologer; Stereology Resource Center). 
A
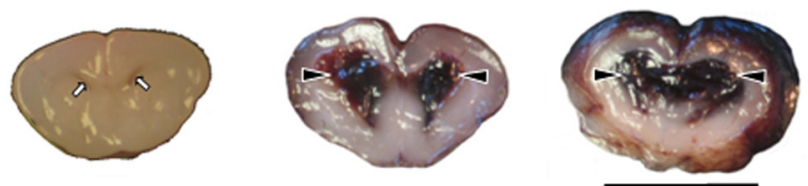

B
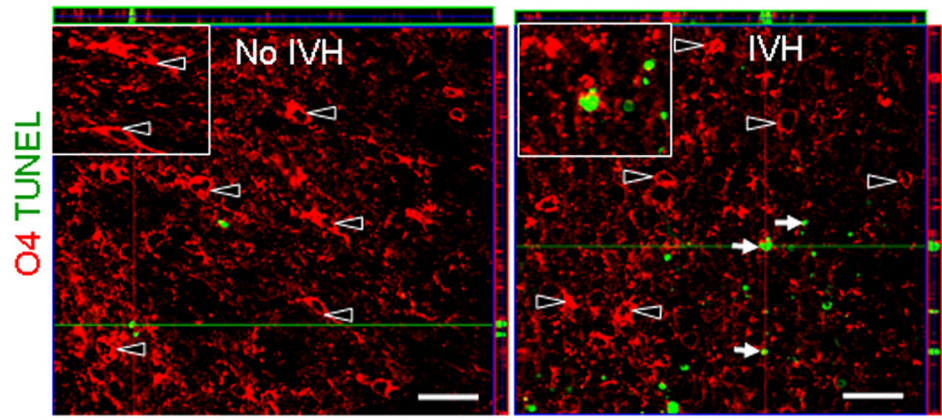

D
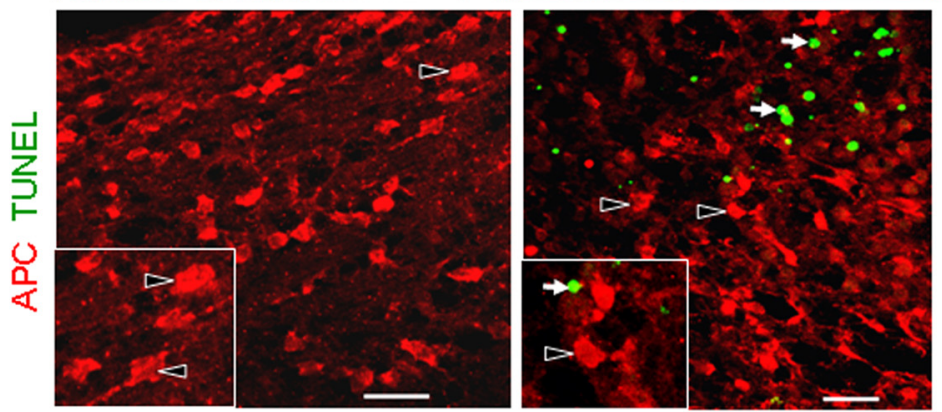

E

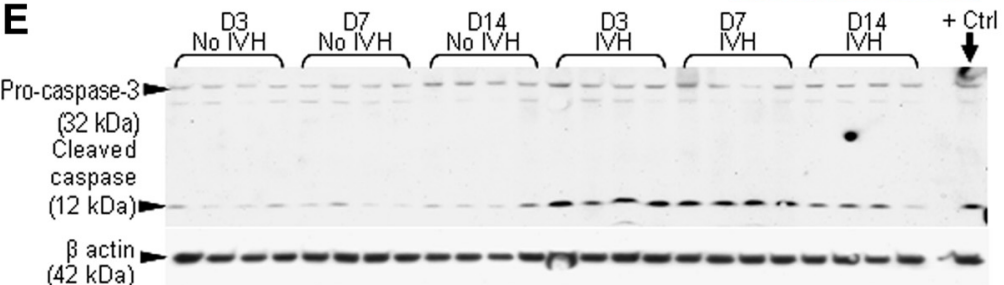

C

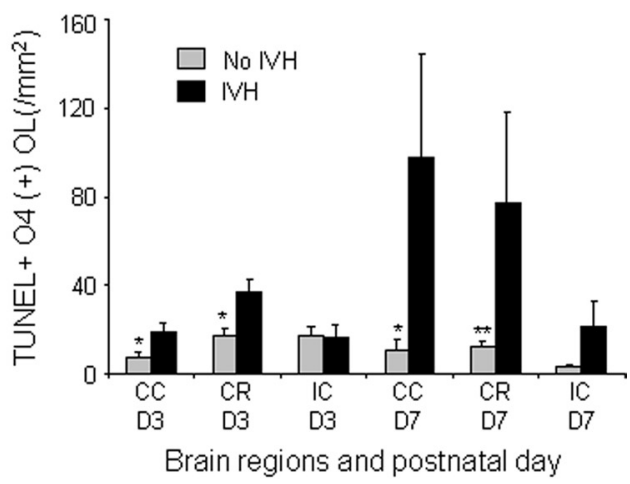

$\mathbf{F}$

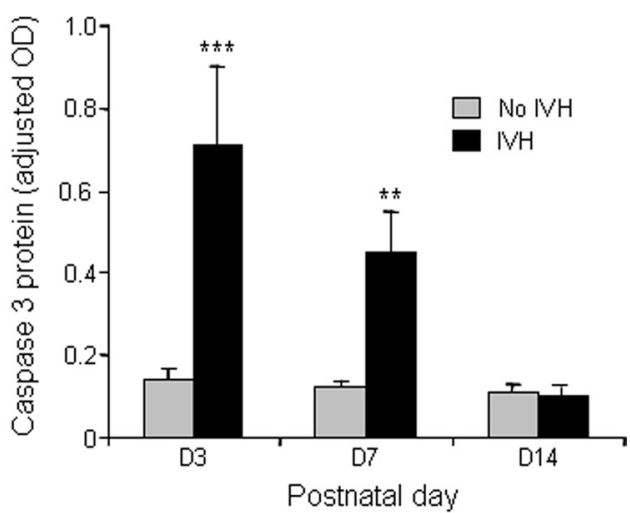

Figure 1. IVH induces apoptosis. $A$, Coronal brain section through frontal lobe of E29 rabbit pup showing normal slit-like ventricle (white arrows, left panel), moderate hemorrhage in the ventricle (arrowheads, middle panel) and severe hemorrhage resulting in fusion of the two ventricles (arrowheads, right panel). Scale bar, $1 \mathrm{~cm} . \boldsymbol{B}$, Representative immunofluorescence of cryosections from 3-d-old E29 pups with and without IVH double labeled for TUNEL and 04 antigen. Above and to the right of the images are orthogonal views in $x-z$ and $y-z$ planes of a composite of $z$-stack of a series of confocal images taken $0.6 \mu \mathrm{m}$ apart. TUNEL $(+)$ nuclei embedded in 04-labeled OL indicate apoptotic OL (arrows). Note apoptotic OL in pups with IVH, but not in controls without IVH. 04 ( + ) OLs are shown (arrowheads). Insets show 04 immunolabeling (left) and apoptotic $0 \mathrm{Ls}$ (right) under high magnification. Scale bars, $25 \mu \mathrm{m}$. C, The bar graph shows mean \pm SEM ( $n=5-6$ each). Apoptotic OLs were more abundant in the corpus callosum (CC) and corona radiata (CR) of pups with IVH pups than glycerol-treated controls without IVH at both days 3 and 7, but not in the internal capsule (IC). D, Representative immunofluorescence of cryosections from 3-d-old E29 pups with and without IVH double labeled for TUNEL- and APC-specific antibody. Note that TUNEL (+) nuclei (arrow) do not overlap with APC (+) OLs (arrowhead). Scale bar, $25 \mu \mathrm{m}$. $\boldsymbol{E}$, Representative Western blot analyses for caspase-3 in the forebrain of premature rabbit pups with and without IVH at postnatal days 3,7 and 14. Each lane represents lysate from whole coronal slice taken at the level of midseptal nucleus of one brain. Values are normalized to $\beta$-actin levels. Ctrl, Control. $\boldsymbol{F}$, Bar chart shows mean $\pm \operatorname{SEM}\left(n=6\right.$ each). Cleaved caspase-3 (active caspase-3, $12 \mathrm{kDa}$ ) levels were higher in pups with than pups without IVH at both day 3 and 7 , unlike day $14 .{ }^{*} p<0.05$, ${ }^{* *} p<0.01$, and ${ }^{* * *} p<0.001$ for the comparison between pups with and pups without IVH.

Fluorescent in situ detection of DNA fragmentation (TUNEL). We performed Fluoro-Jade (Millipore) and TUNEL staining on fixed brain sections as described previously (Georgiadis et al., 2008). For TUNEL staining, $15-\mu \mathrm{m}$-thick tissue sections were air dried on slides, hydrated in $0.01 \mathrm{M}$ PBS, and permeabilized for $5 \mathrm{~min}$ in 1:1 ethanol:acetic acid. An ApopTag-fluorescein in situ DNA fragmentation detection kit (catalog \#S7110; Millipore) was used to visualize TUNEL-labeled nuclei.

Quantification of oligodendrocytes, proliferation, and apoptosis. We counted (1) $\mathrm{O} 4(+)$ and $\mathrm{O} 1(+) \mathrm{OL}$ in double-labeled brain sections, (2) O4 (+) OL colabeled for TUNEL staining and all TUNEL $(+)$ nuclei, $(3)$ cells labeled with both K67 and Olig2 antibodies and all Ki67 (+) cells in double-stained brain sections. From each brain, five coronal sections (20 $\mu \mathrm{m}$ thick) were taken as every third section at the level of midseptal nucleus. Counting was performed in an unbiased fashion and random basis. In the periventricular zone (germinal matrix, caudate nucleus, deep corona radiata, and corpus callosum around the ventricle) neural cell proliferation and in the white matter (corpus callosum, corona radiate and internal capsule) apoptotic OL-, O4-, and O1-positive OL were quantified under confocal microscope using a $60 \times$ lens (Nikon Instruments) by a blinded investigator. We counted objects in $\sim 25$ images ( 5 images $\times 5$ coronal sections) per brain region for each pup $(n=5-6$ pups each group) for every parameter.

Stereological assessment of myelin and astrocytes in the white matter. Unbiased stereology methods, with assistance from a computerized software system (Stereologer; Stereology Resource Center), were used to quantify a range of parameters. Briefly, coronal sections were cut on cryostat at a setting of $30 \mu \mathrm{m}$ thickness with a section sampling interval of three $(90 \mu \mathrm{m})$ to achieve at least six sections at the level of midseptal nucleus. The sections were double labeled with MBP antibody and DAPI (nuclear stain) and quantified as follows. The reference spaces (corona radiata, corpus callosum) were first outlined on the section under $4 \times$ objective. The volume of the outlined area (reference space) was quantified using a point counting probe (frame, $25 \times 25 \mu \mathrm{m}$; guard zone, $2 \mu \mathrm{m}$; interframe interval, $300 \mu \mathrm{m}$ ). The total volume fraction (load) of myelin labeled by antibodies to MBP through a defined reference space was quantified using the object area fraction probe under $60 \times$ oil lens. For 
the area fraction probe (frame, $25 \times 25 \mu \mathrm{m}$; guard zone, $2 \mu \mathrm{m}$; interframe interval, $400 \mu \mathrm{m}$ ), the user clicked on the grid points that touched myelin fibers in sections stained with MBP. The area fraction of myelination was quantified as the ratio of product of the area per point and number of points hitting reference area $\left[\mathrm{a}(\right.$ point $\left.) \cdot \sum P_{\text {ref }}\right]$ over the product of the area per point and number of points hitting the sampled area $\left[\mathrm{a}\right.$ (point) $\left.\cdot \sum P_{\text {samp }}\right]$, as reported recently (Mouton et al., 2009). Sampling continued until the coefficient of error was $<0.10$. To assess gliosis, we performed the following: (1) astrocyte count (labeled against GFAP) using the optical dissector method (frame, $25 \times 25 \mu \mathrm{m}$; guard zone, 2 $\mu \mathrm{m}$; interframe interval, $280 \mu \mathrm{m}$ ); and (2) total volume fraction of astrocyte cell body and glial fibers (Mouton et al., 2009). Volume fraction of astrocytes was quantified in similar fashion as for myelin.

Western blot analyses. We homogenized the frozen brain tissue in sample buffer (3\% SDS, 10\% glycerol, $62.5 \mathrm{mmol}$ of TRIS- $\mathrm{HCl}$, and $100 \mathrm{~mm}$ DTT) using a mechanical homogenizer and boiled the samples immediately for $5 \mathrm{~min}$. We next determined protein concentration in the sample using RC DC protein assay kit (Bio-Rad) and used dilutions of BSA as the standard. Total protein samples were separated by SDS-PAGE according to the previously described method (Ballabh et al., 2007). Equal amounts of protein $(10-20 \mu \mathrm{g})$ were loaded into $4-15 \%$ gradient precast gel (BioRad). The separated proteins were transferred onto PVDF membrane by electro-transfer. The membranes were then incubated with primary antibodies. We detected target proteins with chemiluminescence ECL system (GE Healthcare) by using secondary antibodies conjugated with horseradish peroxidase (Jackson Immunoresearch). We next stripped the blots with stripping buffer (Pierce) and incubated with $\beta$-actin primary antibody followed by secondary antibody and detection with chemiluminescence ECL system. As described previously (Ballabh et al., 2007), the blots from each experiment were densitometrically analyzed using ImageJ. The optical density (OD) values were normalized by taking the ratio of the target protein and $\beta$-actin. The optical density was measured for of all bands together in each lane for both myelin basic protein $(12-32 \mathrm{kDa})$ and myelin-associated glycoprotein (MAG) $(55-65 \mathrm{kDa})$. For BMP4, however, we measured OD of both BMP precursor $(50 \mathrm{kDa})$ and BMP mature from $(23 \mathrm{kDa})$ separately and together.

Quantitative real-time PCR. Quantitative real-time PCR (RT-PCR) was performed as described previously (Ballabh et al., 2007). Briefly, total RNA were isolated from a 1-mm-thick slice taken at the level of midseptal nucleus of the forebrain using Mini RNA isolation kit (Zymo Research). RNA was reverse-transcribed using Superscript II RT (Invitrogen). Real-time reverse transcriptase-PCR was used to analyze mRNA expression using the Stratagene MX3000. Quantification was performed using the efficiencycorrected $\Delta \Delta \mathrm{CT}$ method. The following primers were used for qRT-PCR: BMP2 sense GGTGGAATGACTGGATTG antisense GCATCGAGATAGCACTG; BMP4 (accession \#AF042497) sense TTAACCTCAGCAGCATCC, antisense CAGTCTCGTGTCCAGTAG; Olig1 sense CAGCAGCAGCAACTAAGG, antisense GAGTAGGGCAGGATGACC; Olig2 (accession \#NM_005806) sense 5'-GTGCGGATGCTTATTATAG3', antisense 5'-ATCTGGATGCGATTTGAG-3'; Id2 (NM_002166) sense 5'-AATCCTGCAGCACGTCATCGACTA-3', antisense 5' -TGATGCAGGCTGACAATAGTGGGA-3'; Id4 (accession \#NM_001546) sense 5'-GGCATAATGGCAAATCCTTCAAG-3', antisense 5' -TCACAAGAGATGGGACAGTAGC-3'.

Electron microscopy. We processed brains (day 14) from glyceroltreated pups without IVH, and noggin-treated and vehicle-treated pups with IVH ( $n=3-4$ each). We cut slices ( $2 \mathrm{~mm}$ thickness) from freshly harvested rabbit pup brain using a brain slicer matrix and then dissected the brain slice floating in PBS in a silicone-coated (Slygard; Precision Instruments) Petri dish under a SteReo discovery microscope (Carl Zeiss). The dissected corona radiata and corpus callosum of the white matter were fixed into $2.5 \%$ glutaraldehyde overnight. The tissues were then washed in $0.1 \mathrm{~mol} / \mathrm{L}$ sodium cacodylate buffer, $\mathrm{pH} 7.4$, postfixed in buffered osmium tetroxide for $1-2 \mathrm{~h}$, stained en bloc with $1 \%$ uranyl acetate, dehydrated in graded ethanol solutions, and then embedded in epoxy resin. Sections of 60-90 nm thicknesses were placed onto 200 mesh grids, stained with uranyl acetate and lead citrate, and then were examined with a Techni 12 electron microscope at $80 \mathrm{Kv}$. Digital images were taken using a 16 megapixel Advanced Microscopy Techniques cam-
Table 1. Neurobehavioral evaluation of noggin-treated pups compared to vehicletreated controls with IVH and pups without IVH at postnatal day 14

\begin{tabular}{|c|c|c|c|c|}
\hline System & Test & $\begin{array}{l}\text { No IVH } \\
(n=15)\end{array}$ & $\begin{array}{l}\text { IVH-noggin } \\
(n=14)\end{array}$ & $\begin{array}{l}\text { IVH-vehicle } \\
(n=14)\end{array}$ \\
\hline \multirow[t]{3}{*}{ Cranial nerve } & Aversive response to alcohol & $3(3,3)$ & $3(3,3)$ & $3(3,3)$ \\
\hline & Sucking and swallowing & $3(3,3)$ & $3(3,3)$ & $3(3,3)$ \\
\hline & Vision & $3(3,3)$ & $3(3,3)$ & $3(3,3)$ \\
\hline \multirow[t]{10}{*}{ Motor } & Motor activity & & & \\
\hline & Head & $3(3,3)$ & $3(3,3)$ & $3(3,3)$ \\
\hline & Fore legs & $3(3,3)$ & $3(3,3)$ & $3(3,3)$ \\
\hline & Hind legs & $3(3,3)$ & $3(3,3)$ & $3(2,3)^{*}$ \\
\hline & Righting reflex ${ }^{a}$ & $5(5,5)$ & $5(5,5)$ & $3(3,5)$ \\
\hline & $\begin{array}{l}\text { Locomotion on } 30^{\circ} \text { inclination } \\
\text { Tone } \\
{ }^{c}\end{array}$ & $3(3,3)$ & $3(3,3)$ & $3(2,3)$ \\
\hline & Forelimb & $0(0,0)$ & $0(0,0)$ & $0(0,0)$ \\
\hline & Hindlimb & $0(0,0)$ & $0(0,0)$ & $0(0,0)$ \\
\hline & $\begin{array}{l}\text { Hold their position at } 60^{\circ} \\
\text { inclination (latency to slip } \\
\text { down the slope in seconds) }\end{array}$ & 13.1 & 15.3 & $10.9^{* *}$ \\
\hline & Distance walked in $60 \mathrm{~s}$, inches & 101 & 103 & $68^{* * *}$ \\
\hline Gait $^{d}$ & & $4(4,4)$ & $4(4,4)$ & $3.5(2.0,4)^{* * * *}$ \\
\hline Motor impairment & Weakness in extremities, $\%$ & $0 \%$ & $7.1 \%$ & $28.6 \%$ \\
\hline \multirow[t]{2}{*}{ Sensory } & Facial touch & $3(3,3)$ & $3(3,3)$ & $3(3,3)$ \\
\hline & Pain & $3(3,3)$ & $3(3,3)$ & $3(3,3)$ \\
\hline
\end{tabular}

Values are median and interquartile range. Zero is the worst response, and 3 is the best response. ${ }^{a}$ Score (range, 1-5): $n$ o. of times turns prone within $2 \mathrm{~s}$ when placed in supine out of five tries.

${ }^{b}$ Score (range, $0-3$ ): 0 , does not walk; 1 , takes a few steps (less than 8 inches); 2 , walks for $9-18$ inches; 3 , walks very well beyond 18 inches.

'Score (range, 1-3): 0 , no increase in tone; 1 , slight increase in tone; 2 , considerable increase in tone; 3 , limb rigid in flexion or extension.

${ }^{d}$ Gait was graded as 0 (no locomotion), 1 (crawls with trunk touching the ground for few steps and then rolls over), 2 (walks taking alternate steps, trunk low and cannot walk on inclined surface), 3 (walks taking alternate steps, cannot propel its body using synchronously the hind legs, but walks on $30^{\circ}$ inclined surface), 4 (walks, runs, and jumps without restriction, propels the body using synchronously the back legs, but limitation in speed, balance, and coordination manifesting as clumsiness in gait), or 5 (normal walking).

${ }^{*} p=0.03,{ }^{* *} p=0.05,{ }^{* * *} p=0.039,{ }^{* * * *} p=0.04$ for the comparison between IVH- and noggin-treated IVH pups.

era. We acquired 12-20 images per brain region for a total of $\sim 270$ images ( 3 groups $\times 3-4$ brain each group $\times 2$ brain regions $\times 12-20$ images $=270$ images). Electron micrographs were evaluated for myelinated axons per unit area; and g-ratio (ratio of axonal diameter with myelin sheath and axonal diameter without myelin sheath) of myelinated axons in the 3 groups of pups were determined using image processing software, ImageJ (NIH).

Neurobehavioral assessment. We performed neurobehavioral assessment at postnatal day 14 based on the previously described scoring protocol (Georgiadis et al., 2008; Chua et al., 2009). The evaluation was performed by two physicians, who were blinded to the group assignment. We tested cranial nerves by testing smell (aversive response to ethanol), sucking and swallowing (formula delivered by a plastic pipette). We scored the responses on a scale of $0-3,0$ being the worst response and 3 the best. Motor evaluation included tone (modified Ashworth's scale), motor activity, locomotion at $30^{\circ}$ angle, righting reflex and gait. Tone was examined by active flexion and extension of forelegs and hind legs (score $0-3$ ). The righting reflex was evaluated by the ability and rapidity to turn prone when placed in supine position. Sensory evaluation included touch on face (touching face with cotton swab) and extremities as well as pain on limbs (mild pin prick). Grading of tone, gait, and locomotion at $30^{\circ}$ angle are illustrated in the footnote of Table 1 . To test coordination and muscle strength in forelegs and hind legs, we evaluated the ability of the pups to hold their position on a ramp pitched at a slope of $60^{\circ}$. The test was conducted on a rectangular surface $(18 \times 6$ inch $)$ kept at $60^{\circ}$ inclination. We placed the pup at the upper end of the inclination and measured the latency to slip down the slope. We performed the visual cliff test to assess the vision. All animals could detect the cliff.

Statistics and analysis. Data are expressed as means and SEM. To determine differences in the apoptosis, proliferation, caspase- 3 activity between rabbit pups with and without IVH, two-way ANOVA was used. 
The independent factors in two-way ANOVA were postnatal age ( $\mathrm{d} 3 \mathrm{vs} \mathrm{d} 7)$ and presence of IVH treatment (IVH vs no IVH). To assess differences in density of pre-OLs and immature OLs, two-way ANOVA with repeated measures was used. The repeated measures were applied to the three white matter regions - corona radiata, corpus callosum, and internal capsule. For Western blot analyses data, four group comparisons were done using one-way ANOVA. All post hoc comparisons to test for differences between means were done using Tukey multiple-comparison test at the 0.05 significance level.

\section{Results}

IVH induces apoptosis and suppresses proliferation of OL progenitors

As IVH results in neural cell death (Georgiadis et al., 2008) and influences neural cell proliferation (Xue et al., 2003), we investigated the relative contributions of cell death and cell proliferation to the total pool of OLs at successive time intervals after IVH in our rabbit pup model (Ballabh et al., 2007; Georgiadis et al., 2008; Chua et al., 2009). Double labeling of the brain sections for TUNEL and O4 antigen showed that the apoptosis of $\mathrm{O} 4(+)$ cells was significantly more abundant in the corpus callosum and corona radiata of pups with IVH compared with glyceroltreated controls without IVH at both postnatal days $3(p=0.032$ and 0.04$)$ and $7(p<0.05$ and 0.007$)$ (Fig. $1 B, C)$. In the internal capsule, however, the density of apoptotic O4 $(+)$ OLs and also other neural cells did not differ between pups with and without IVH. The absence of substantial cell death in the internal capsule, unlike the other white matter regions, was attributed to the distant anatomical location of this brain region from the blood filled ventricle. Accordingly, density of all TUNEL-positive cells in the corona radiata and corpus callosum were also higher at days 3, 7, and 14 in pups with IVH compared with pups without IVH $(p<0.05$ each, data not shown). Because $\mathrm{O} 4$ labeling at day 14 was inconsistent and variable in the white matter regions, the evaluation of $\mathrm{O} 4$-positive cell death at this age was excluded from the present study. We next tested apoptosis of mature OLs by combining TUNEL staining with APC (CC1 clone) immunolabeling. We found that apoptotic APC $(+)$ OLs were relatively scarce $\left(2.7 \pm 1.2 / \mathrm{mm}^{2}\right.$ vs none in pups with and without IVH) in pups with IVH (Fig. $1 D)$.

To further confirm apoptotic activity in the forebrain of pups with IVH as a function of postnatal age, we measured caspase-3 expression at postnatal days 3,7 , and 14 by Western blot analysis (Fig. 1E). The results showed that the expression of cleaved capsase-3 (active caspase-3, $12 \mathrm{kDa}$ ) was higher in pups with IVH compared with controls without IVH (glycerol treated) at both days 3 and 7 ( $p=0.001$ and 0.022 ), but not at day 14 ( $p=0.96)$ (Fig. $1 F)$. The findings indicate that apoptotic cell death in IVH pups might be caspase dependent at both days 3 and 7 .

We next assessed proliferation of OL progenitors in the pups with and without IVH (glycerol-treated). As OL progenitors in the white matter originate from the VZ and SVZ of human fetuses (17-23 weeks) and since terminally differentiated OL typically do
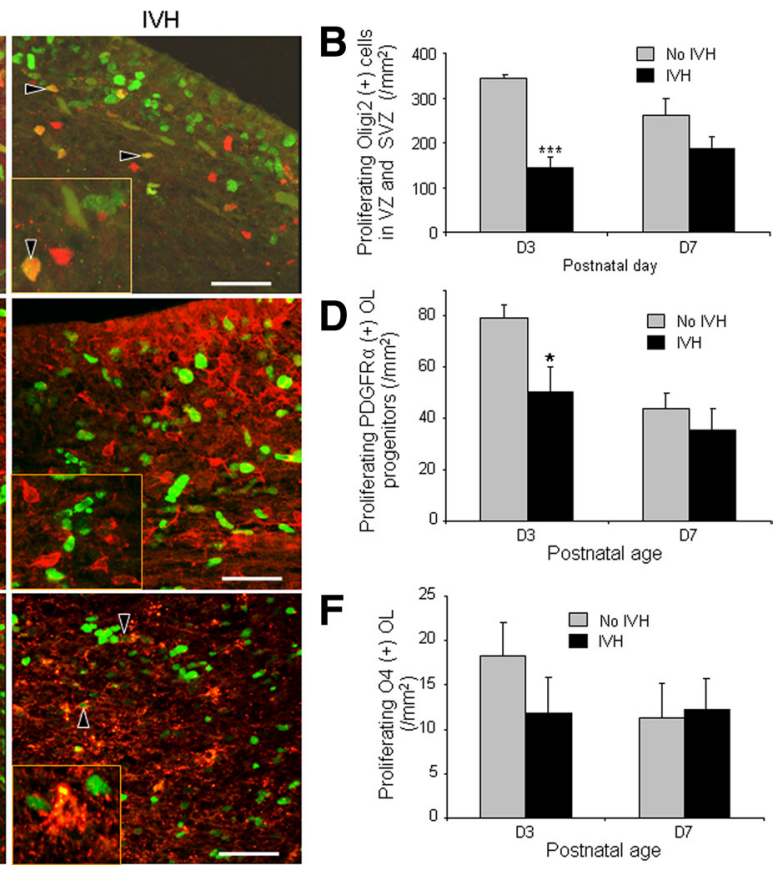

Figure 2. IVH suppresses proliferation of oligodendrocyte progenitors. $A$, Cryosections from the forebrain of rabbit pups were labeled with $(+)$ cells was comparable in pups with and without IVH. Insets show immunolabeling under high magnification. Scale bar, $50 \mu \mathrm{m} .{ }^{*} p<$ $0.05^{* * *} p<0.001$ for the comparison between pups with and without IVH.

not proliferate after completion of their migration (Rakic and Zecevic, 2003), we assessed proliferation of all neural cells and OL progenitors in the VZ and SVZ at both days 3 and 7 (Fig. 2). To label OL progenitors, we used Olig2-, PDGFR $\alpha$-, and O4-specific antibodies. Olig2 is expressed by both OL progenitors and mature OL, PDGFR $\alpha$ antibody identifies early OL progenitors, and O4 antibody labels both late progenitors and other OLs. Double labeling of the brain sections with Olig-2 and Ki67 (proliferation marker) antibodies showed that the density of proliferating Olig2 $(+)$ cells was reduced in pups with IVH than pups without IVH (glycerol treated) at postnatal days $3(p<0.001)$, but not at day 7 (Fig. 2A,B). Accordingly, density of proliferating PDGFR $\alpha$ positive OLs was less in pups with IVH compared with pups without IVH at day 3 ( $p<0.05$ ), but not at day 7 (Fig. $2 C, D$ ). However, mitotically active O4-positive OLs were comparable between pups with and without IVH at both days 3 and 7 (Fig. $2 E, F)$. In addition, the development of IVH significantly suppressed the proliferation of all neural cells (all Ki67 + cells) in the lateral VZ and SVZ at both postnatal days 3 and $7(p<0.001$ each). Proliferating OL in the white matter were scarce at days 3 and 7. Together, IVH-induced apoptotic cell death in the periventricular white matter and a transient suppression in the proliferation of OL progenitors in the VZ and SVZ.

\section{IVH arrests maturation of oligodendrocyte lineage in preoligodendrocyte stage}

The findings that IVH-induced apoptosis and inhibited the proliferation of periventricular OL progenitors led us to the question 
A
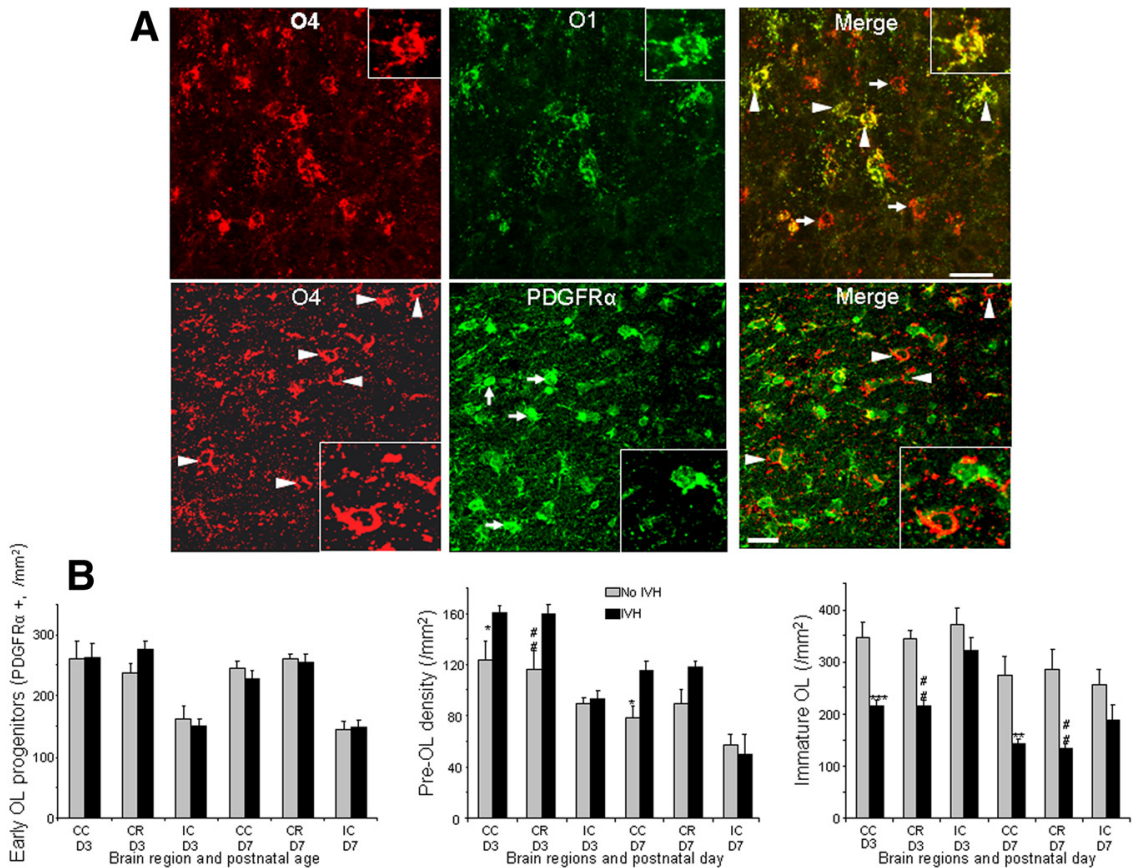

Figure 3. IVH induces maturation arrest of pre- $0 \mathrm{~L}$ and affects 0 lig 2, SOX10, and Id 4 transcription factors. $\boldsymbol{A}$, Representative double immunolabeling of the cryosections from the forebrain of pups with IVH using a combination of 04 with 01 or PDGFR $\alpha$ antibody at day 3. Note that pre-0Ls are labeled with 04, not with 01 antibody (arrows), and immature $0 \mathrm{~L}$ are labeled with both 04 and 01 (arrowheads). Note that 04 (+) cells (arrowhead) mostly do not overlap PDGFR $\alpha(+)$ ) 0 (arrow). Insets show 04, 01, and PDGFR $\alpha$ immunolabeling under high magnification. Scale bar, $25 \mu \mathrm{m}$. $\boldsymbol{B}$, The data in the bar charts represent mean \pm SEM ( $n=5-6$ each). At both day 3 and 7, the density of PDGFR $\alpha(+)$ OL was comparable between pups with and without IVH in corpus callosum (CC), corona radiata (CR), and internal capsule (IC). However, the pre-0 Ls $(04+01-)$ were more abundant in the $C(\mathrm{C}$ and CR of pups with IVH than without IVH at day 3, but not in the IC. At day 7, pre-0Ls were more in number in the CC of IVH pups relative to pups without IVH, but not in the $\mathrm{CR}$ and IC. The density of immature $\mathrm{LL}(04+01+)$ was significantly reduced in rabbit pups with IVH than in pups without IVH at both day 3 and 7 in the corpus callosum and corona radiata, unlike the internal capsule. ${ }^{* * *} p<$ $0.001,{ }^{* *} p<0.01$, and ${ }^{*} p<0.05$ for the comparison between pups with and without IVH in corpus callosum. ${ }^{\#} p<0.05$ for the comparison between pups with and without IVH in corona radiata.

of whether the density of early OL progenitors (PDGFR $\alpha$ ), pre-OLs (O4+ and O1-) and immature OLs $(\mathrm{O} 4+$ and $\mathrm{O} 1+)$ differed in the white matter regions of premature pups with IVH compared with controls (glycerol-treated) without IVH. To this end, we labeled the brain sections with $\operatorname{PDGFR} \alpha, \mathrm{O} 4$ (biotinylated)-, and O1-specific antibodies (Fig. 3A). We found that early OL progenitors (PDGFR $\alpha+$ ) were comparable between pups with and without IVH in the white matter regions at both days 3 and 7 (Fig. 3B). However, the density of pre-OLs was higher in the corpus callosum and corona radiata by $\sim 20 \%$ ( $p=$ 0.017 and 0.006 ), but not in the internal capsule, of pups with IVH compared with pups without IVH at day 3 (Fig. 3B). At day 7 , pre-OLs were also more abundant in the corpus callosum $(p=$ 0.029 ) of pups with IVH relative to pups without IVH, but not in the corona radiata and internal capsule $(p=0.8$ and 0.6$)$ (Fig. $3 B$ ). More importantly, the density of immature $\mathrm{OL}(\mathrm{O} 4+\mathrm{O} 1+)$ was significantly reduced in rabbit pups with IVH compared with pups without IVH at both days $3(p<0.001,0.002)$ and $7(p=$ $0.007,0.003)$ in the corpus callosum and corona radiata, unlike the internal capsule ( $p=0.25,0.15)$ (Fig. 3B). A difference in the impact of IVH on the maturational changes in the pre-OLs and immature OLs among the white matter regions was ascribed to their location relative to the ventricle. Collectively, the data suggested that arrested maturation of OL lineage at the pre-OL stage in pups with IVH resulted in two histological changes-reduction in the density of immature OLs (myelinating OLs) and the resultant abundance of pre-OL (nonmyelinating OL).
IVH induces distinct changes in bHLH transcription factors

While Olig1, Olig2, and Sox10 favor OL maturation, Id 2 and Id 4 have inhibitory influences (Nicolay et al., 2007). We assessed expression of these factors in a coronal slice at the level of midseptal nucleus in pups with and without IVH (glycerol treated) at postnatal days $1,3,7$, and 14 by real-time quantitative PCR (Fig. 4A). Olig2 expression was significantly reduced in pups with IVH relative to pups without IVH at days 3,7 , and 14 ( $p=0.002,0.025$, and 0.007 , respectively), but not at day 1 $(p=0.137)$. Olig1 expression, albeit low in IVH pups, was not significantly reduced in pups with IVH compared with those of controls at any of the postnatal days. Sox10 expression was substantially reduced in pups with IVH relative to controls at days $1,3,7$, and 14 ( $p=0.008$, $0.001,0.002$, and 0.025 , respectively). Conversely, Id4 mRNA expression was significantly elevated in pups with IVH compared with controls without IVH at days 1 and $3(p<0.001,0.004)$, but not at days 7 and $14(p=0.09,0.8)$. Id 2 mRNA accumulation was also higher in pups with IVH compared with controls at day 1 $(p<0.02)$, but not at days 3,7 , and 14 ( $p>0.4$ at all time points).

We next measured protein levels of Olig1 and Olig2 by Western blot analyses. Consistent with mRNA expression, Olig2 levels were less in pups with IVH compared with pups without IVH at days 7 and 14, but not at day 3 (Fig. 4B). Olig1 levels were comparable between pups with and without IVH. A reduction in the expression of Olig2, but not Olig1, in IVH is consistent with the previous reports on animal models of acute brain injury (Buffo et al., 2005). Together, induction of IVH elevated inhibitory and suppressed activating transcription factors involved in the specification and maturation of OL lineage, consistent with arrested maturation of pre-OLs in the previous experiment.

\section{High bone morphogenetic protein levels in premature rabbits with IVH}

BMP overexpression diverts OL progenitors to astrocyte lineage, promotes astrocytosis, and inhibits OL differentiation by downregulating Olig1 and Olig2 transcription factors (Nicolay et al., 2007). Therefore, we assessed BMP2 and BMP4 levels in the forebrain of rabbit pups with and without IVH. BMP2 mRNA accumulation was significantly higher in pups with IVH than glycerol-treated controls without IVH at day 3,7 , and 14 ( $p=$ $0.05,0.002,0.001)$, but not at day $1(p=0.8)$ (Fig. $5 A)$. In addition, the $B M P 2$ level increased as a function of postnatal age in pups with IVH $(p<0.001)$, but not in controls without IVH. However, Western blot analysis revealed that BMP2 protein (precursor: $60 \mathrm{kDa}$, mature: $20 \mathrm{kDa}$ ) levels were comparable between pups with and without IVH (Fig. 5B). Accordingly, immunohistochemistry showed that BMP2 immunoreactivity was comparable between pups with and without IVH. BMP2 was weakly expressed in the ependyma and Tuj1 (+) neuronal progenitors of 

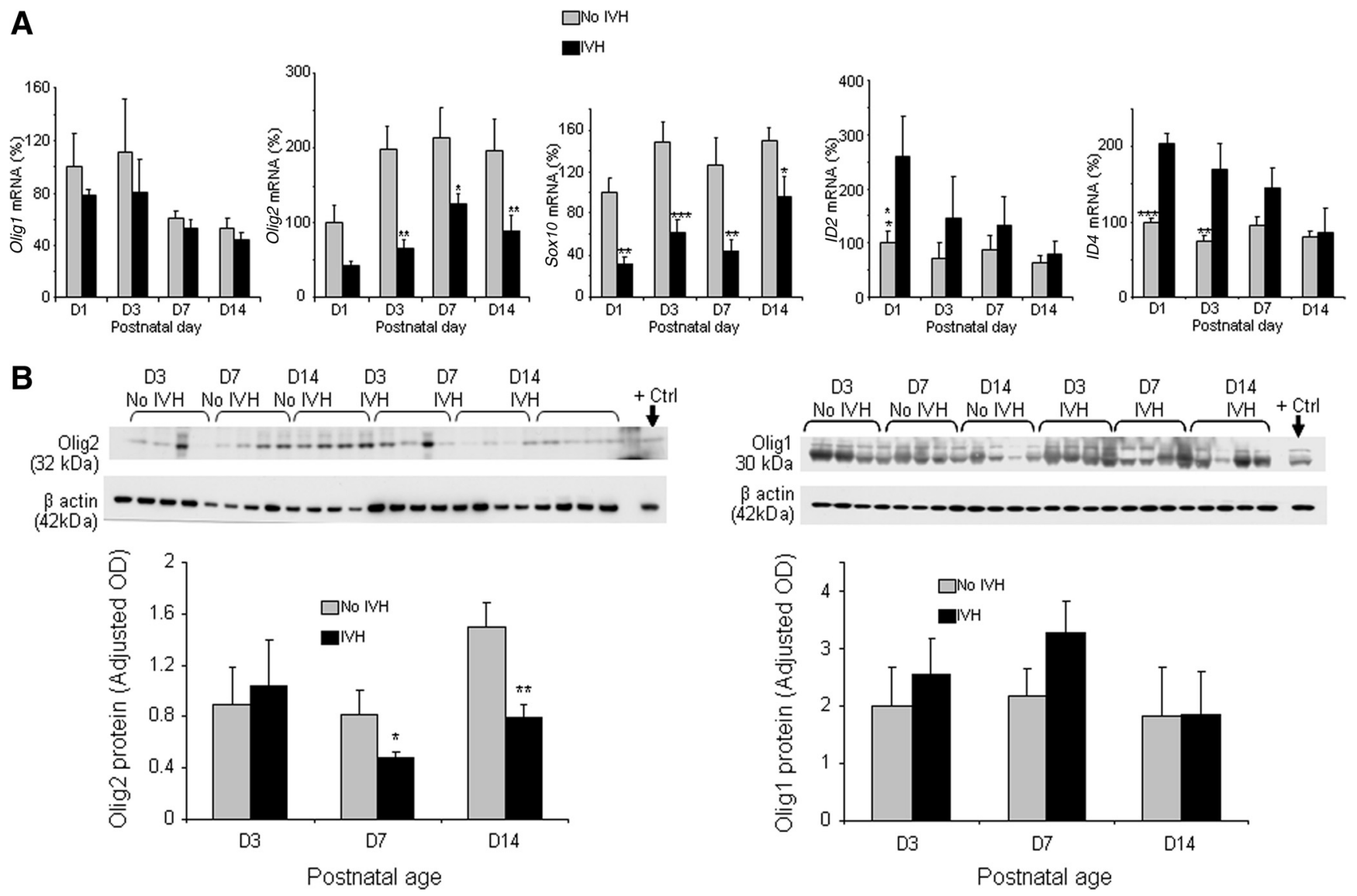

Figure 4. IVH distinctly affects the bHLH transcription factors. $A$, The data are mean \pm SEM ( $n=6$ at each time point). Olig1 gene expression was not different in pups with and without IVH. Olig2 expression was significantly lower in pups with IVH than in pups without IVH at days 3,7 , and 14, but not at day 1. Sox10 expression was significantly lower in pups with IVH than in pups without IVH on all days. Id2 mRNA levels were higher in pups with IVH than pups without IVH at day 1, but not on other days. Id4 mRNA expression was significantly greater in pups with IVH compared with pups without IVH at days 1 and 3 , but not at day 7 and $14 .{ }^{* * *} p<0.001$, ${ }^{* *} p<0.01$, and ${ }^{*} p<0.05$ for the comparison between pups with and without IVH. $\boldsymbol{B}$, Representative Western blot analyses for Olig2 and Olig1 in the forebrain of premature rabbit pups with and without IVH at postnatal days 3, 7, and 14. Each lane represents lysate from whole coronal slice taken at the level of midseptal nucleus of one brain. Rat brain was taken as positive control. Values are normalized to $\beta$-actin levels. Bar chart shows mean \pm SEM ( $n=6$ each). Olig2 levels were significantly lower in rabbit pups with IVH compared with controls at days 7 and 14 , but not at day 3 . However, Olig 1 levels were comparable between pups with and without IVH at days 3,7 , and 14 . ${ }^{* *} p<0.01$ and ${ }^{*} p<0.05$ for the comparison between pups with and without IVH.

the SVZ. However, BMP2 was strongly expressed in cortical neurons particularly in neuropils of the cerebral cortex (Fig. 5C). The discrepancy between protein and mRNA abundance of BMP2 in pups with IVH can be attributed to either post-transcriptional or post-translational factors. Accordingly, BMP2 levels were similar in human premature infants with and without IVH in the three brain regions - cortex, white matter and germinal matrix - on Western blot analysis (Fig. 5D).

Real time PCR revealed that BMP4 mRNA expression was greater in pups with IVH relative to controls at days 3,7 and 14 $(p<0.003,0.001$, and 0.001 respectively), but not at day 1 ( $p=$ 0.646) (Fig. 6A). BMP4 levels also increased with the advancing postnatal age in pups with IVH, unlike controls $(p<0.001)$. Consistent with mRNA levels, the expression of BMP4 protein [both precursor $(50 \mathrm{kDa})$ and mature $(23 \mathrm{kDa})$ form] measured by Western blot analyses, was higher in pups with IVH than in glycerol-treated controls without IVH at days 3,7 and 14 ( $p=$ $0.044,0.001$, and 0.012) (Fig. 6B). The levels of mature BMP4 form $(23 \mathrm{kDa})$ alone were also significantly higher in pups with IVH compared with controls at day 7 and $14(p=0.02$ and 0.017 ), but not at day 3. Immunostaining showed that BMP4 expression was more abundant in the VZ, SVZ, and adjacent white matter of rabbit pups with IVH compared with controls without IVH (Fig. 6C). BMP4 was strongly expressed in Tuj1 $(+)$ neuronal precursors of the SVZ, MAP2 $(+)$ neurons of the cerebral cortex and white matter, as well as APC $(+)$ OL in the white matter. The immunoreactivity on these neural cells were stronger in pups with IVH than controls without IVH. BMP4 immunoreactivity was weak-to-absent in the glial fibrillary acidic protein (GFAP)-positive astrocytes. Together, IVH-induced upregulation of BMP4 in premature rabbit pups.

\section{High bone morphogenetic protein expression in premature humans with IVH}

To determine whether human premature infants with IVH develop similar elevation in BMP4 secondary to IVH as those of rabbit pups, we evaluated postmortem human materials for BMP4 expression. Immunolabeling revealed that BMP4 expression was more abundant in the VZ, SVZ, and the subjacent white matter of infants with IVH compared with controls without IVH (Fig. 7A). Immunoreactivity to BMP4 was noted in the Tuj1 (+) neuronal precursors of the SVZ, MAP2 $(+)$ neurons of the cortex, Olig2 $(+)$ and NG2 $(+)$ cells of the white matter and SVZ (Fig. 7A). BMP4 was moderately expressed by GFAP $(+)$ astrocytes in SVZ and adjacent white matter of premature infants of 23-26 week with IVH, and weakly in this groups of infants with- 
A
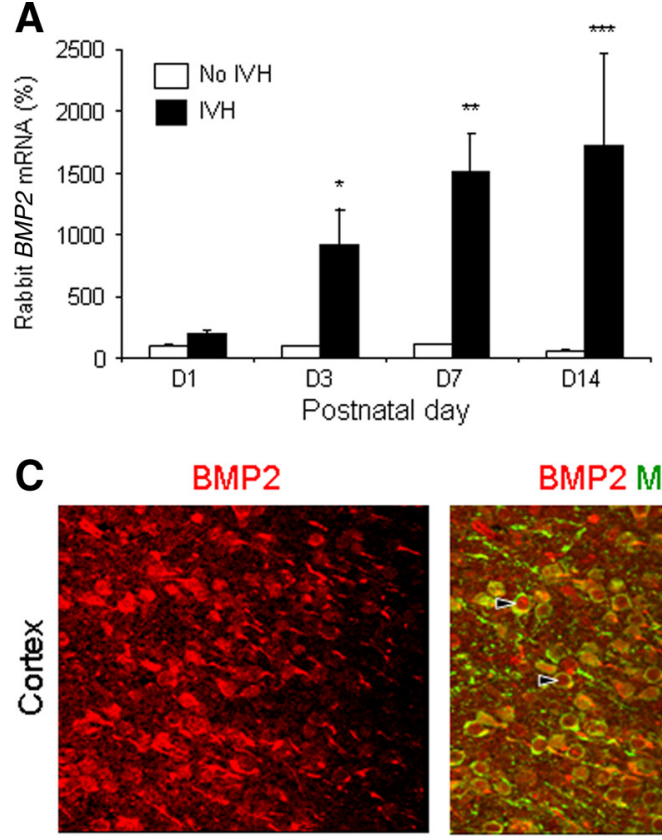

BMP2

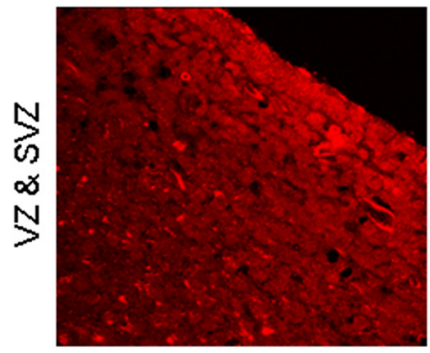

B
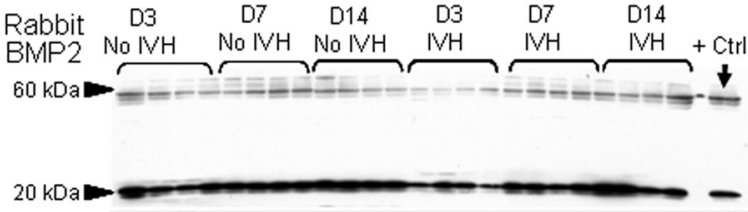

$\beta$ actin - - - - - - - - - -

(42 kDa)

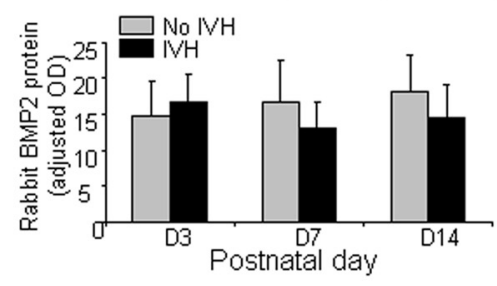

D
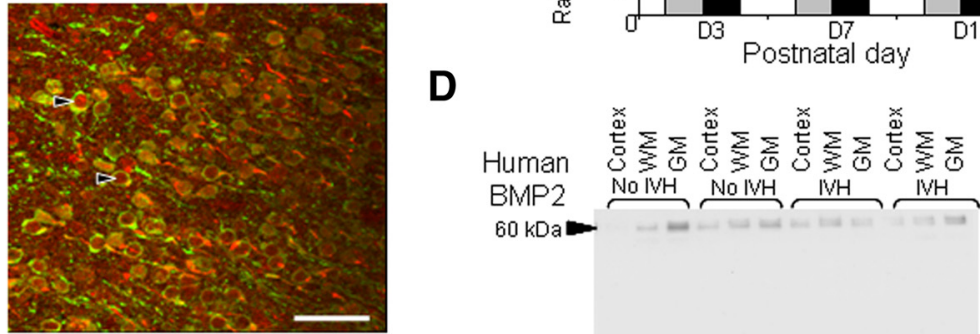

$60 \mathrm{kDa}$
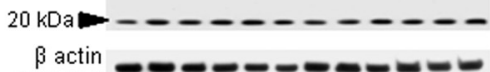

$(42 \mathrm{kDa})$

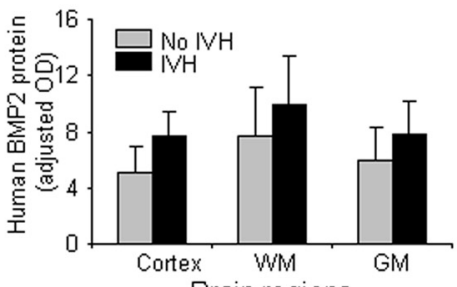

Figure 5. Effect of IVH on BMP2 levels. $A$, The data are mean \pm SEM ( $n=6$ each time point). BMP2 mRNA accumulation was significantly higher in pups with IVH than glycerol-treated pups without IVH at days 3, 7, and 14, but not at day 1. In addition, BMP2 level increased as a function of postnatal age in pups with IVH, but in controls without IVH. $\boldsymbol{B}$, Representative Western blot analyses for BMP2 in premature rabbit pups with and without IVH at postnatal days 3, 7, and 14 . Rat brain was used as positive control (Ctrl). Each lane represents lysate from whole coronal slice taken at the level of midseptal nucleus of one brain. Values are normalized to $\beta$-actin levels. The bars are mean \pm SEM ( $n=6$ each time point). BMP2 expression was comparable in rabbit pups with IVH and glycerol-treated controls without IVH on days 3, 7, and 14. C, Representative immunofluorescence of cryosections from a 3-d-old pup labeled with a combination of BMP2 with Tuj1- or MAP-2specific antibody. BMP2 was weakly expressed in the ependyma and Tuj-1 $(+)$ neuronal precursor cells of SVZ. In the cerebral cortex, BMP2 immunoreactivity was noted in MAP2 $(+)$ neurons (arrowheads). Scale bar, $50 \mu \mathrm{m}$. D, Representative Western blot analyses for BMP2 in human premature infants with and without IVH. Values are normalized to $\beta$-actin levels. The bars are mean \pm SEM ( $n=6$ each time point). BMP2 expression in the germinal matrix (GM) (VZ and SVZ), white matter (WM), and cerebral cortex was comparable in infants with and without IVH.

out IVH. Western blot analyses revealed that BMP4 protein [both precursor $(50 \mathrm{kDa})$ and mature $(23 \mathrm{kDa})$ form] levels were significantly higher in the VZ and SVZ (germinal matrix, $p=0.048$ ) as well as in the white matter $(p<0.014)$ of the infants with IVH relative to infants without IVH, but not in the cerebral cortex $(p=0.07)$ (Fig. $7 B)$. The levels of mature BMP4 form $(23 \mathrm{kDa})$ alone were also significantly elevated in white matter of premature infants with IVH compared with infants without IVH $(p<$ $0.02)$, but not in the germinal matrix $(p=0.07)$ and cerebral cortex $(p=0.12)$. In contrast to BMP4, BMP2 protein levels were similar in the cortex, white matter, and germinal matrix of premature infants with and without IVH (data not shown). Together, the data suggested that the development of IVH triggered upregulation of BMP4 in the forebrain of premature infants just as in the rabbit pups.

Intracerbroventricular infusion of noggin alleviates neurological impairment

To determine whether noggin treatment attenuates neurological impairment, we performed a neurobehavioral evaluation among three sets of premature rabbit pups at postnatal day 14 based on our previously described scoring system (Chua et al., 2009): (1) glycerol-treated pups without IVH; (2) pups with IVH treated with ICV noggin; and (3) pups with IVH treated with ICV vehicle (Table 1). The severity of IVH, measured by head ultrasound, in noggin-treated pups was similar to those of vehicle-treated controls. The seven day course of noggin or vehicle treatment was initiated at $24 \mathrm{~h}$ age because BMP levels were elevated in pups with IVH at all days-3, 7 and 14 and since maturation arrest of OLs was noted at both days 3 and 7 .

We noted significant weakness in the forelegs of one and the hind legs of three vehicle-treated pups with IVH (28.6\%), whereas one pup in the noggin-treated group (7\%) had weakness in the left hind-leg manifesting adduction, internal rotation of the back leg and asymmetry in gait. The average scores for gait were substantially better in noggin-treated pups than in vehicletreated controls $(p=0.04)$. The motor activity of the back legscapability to lift the trunk and pelvis-was also better in treated pups compared with vehicle controls $(p<0.03)$. The mean distance walked in $60 \mathrm{~s}$ was longer in noggin-treated pups relative to 

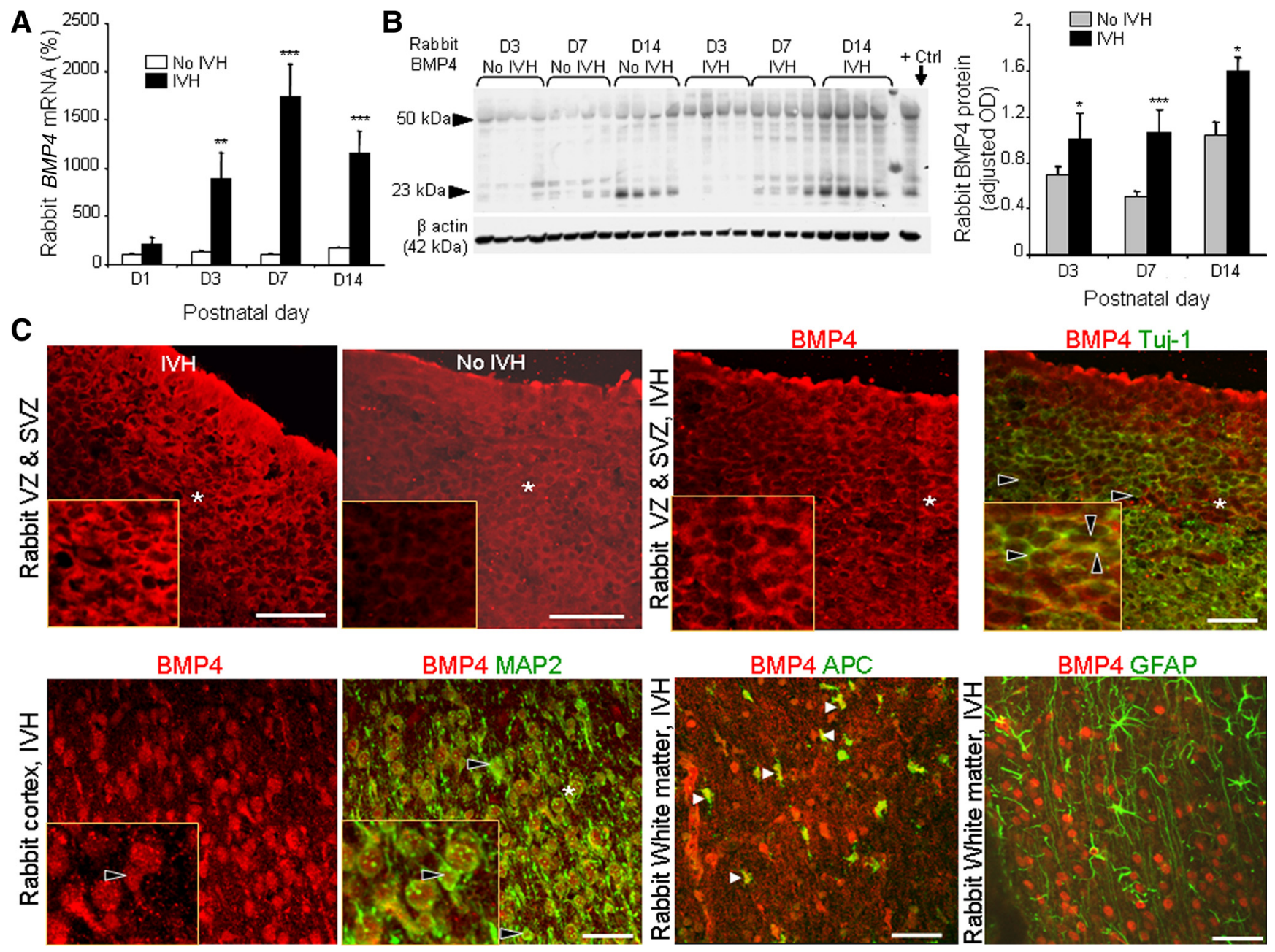

Figure 6. IVH induces elevation of BMP4 in rabbit pups. $A$, The data are mean \pm SEM ( $n=6$ each time point). Both BMP4 mRNA accumulation was significantly higher in pups with IVH than glycerol-treated pups without IVH at days 3, 7, and 14, but not at day 1. In addition, BMP4 levels increased as a function of postnatal age in pups with IVH, but not in controls without IVH. $\boldsymbol{B}$, Representative Western blot analyses for BMP4 in premature rabbit pups with and without IVH at postnatal 3, 7, and 14. 293T whole-cell lysate (sc-113395; Santa Cruz Biotechnology) was used as positive control. Each lane represents lysate from whole coronal slice taken at the level of midseptal nucleus of one brain. Values are normalized to $\beta$-actin levels. Error bars are mean \pm SEM ( $n=$ 6 each time point). BMP4 expression [both precursor $(50 \mathrm{kDa})$ and mature $(23 \mathrm{kDa})$ together] was higher in rabbit pups with IVH than in glycerol-treated controls without IVH on days 3,7 , and 14 . C, Representative immunofluorescence of cryosections from 3-d-old E29 pups with and without IVH labeled with BMP4 antibody (top). All insets show high-magnification views from the region in the image indicated by asterisks. Immunoreactivity to BMP4 is more abundant in IVH subjects than controls without IVH. Cryosections are shown from rabbit forebrain (E29 pup of $3 \mathrm{~d}$ postnatal age) double labeled with a combination of BMP4 with Tuj1, MAP2, APC, or GFAP antibodies. BMP4 was expressed by Tuj1-positive neuronal precursors (arrowheads) of the VZ and SVZ, MAP2-positive neurons (arrowheads) of the cerebral cortex and APC-positive oligodendrocytes (arrowheads) in the white matter. However, BMP4 immunoreactivity was absent on GFAP-positive astrocytes. Scale bar, $50 \mu \mathrm{m}$.

vehicle controls ( $p=0.039$ ). The latency to slip down a ramp pitched at a slope of $60^{\circ}$ was substantially longer in duration in noggin-treated pups compared with vehicle controls $(p<0.05)$. The tone in the back legs of two pups with motor impairment in the vehicle-treated group was slightly increased $($ score $=1$ ). No differences were noted in sensory and cranial nerves in the three sets of rabbit pups. Importantly, we did not observe any apparent adverse effect attributable to noggin treatment among pups with IVH receiving this medication. Together, noggin-treated pups with IVH exhibited better neurobehavioral score than vehicletreated controls with IVH.

\section{Noggin treatment blocks BMP signaling, enhances myelination}

To confirm the blocking of BMP signaling by noggin treatment, we measured phospho-Smad1 (Ser463)/Smad5 (Ser463/465)/ Smad8 (Ser426/428) levels in the forebrain of (1) noggin-treated pups with IVH, (2) vehicle-treated pups with IVH, and (3) pups without IVH (glycerol treated). We found that phospho-Smad1/ $5 / 8$ levels, measured by Western blot analyses, were significantly elevated in rabbit pups with IVH compared with controls without IVH $(p<0.001)$, suggesting enhanced BMP signaling in rabbit pups with IVH. Importantly, noggin treatment significantly reduced phosho-Smad $1 / 5 / 8$ in rabbit pups with IVH $(p=$ 0.001) (Fig. 8A). Hence, the induction of IVH enhanced BMP signaling; and noggin treatment blocked BMP signaling, which was consistent with the previous report (Cate et al., 2010).

We next assessed expression of MBP in four sets of P14 rabbit pups: (1) glycerol-treated pups without IVH; (2) glycerol-treated pups with IVH; (3) ICV noggin-treated pups with IVH; and (4) ICV vehicle-treated pups with IVH. We performed stereological quantification of myelin on brain sections stained with MBP antibody and Western blot analyses on homogenates from a brain slice taken from the forebrain at the level of midseptal nucleus. Stereological analyses showed that the volume fraction (load) for MBP in the corpus callosum was higher in noggin-treated pups 


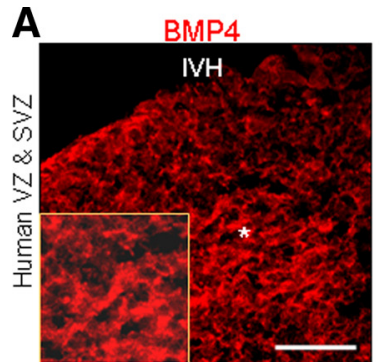

$\mathrm{BMP} 4$

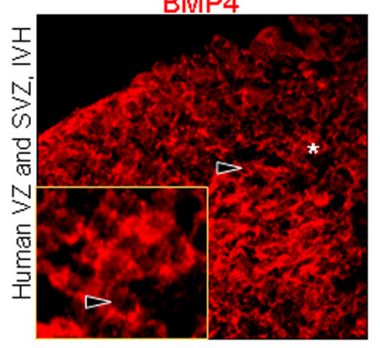

BMP4

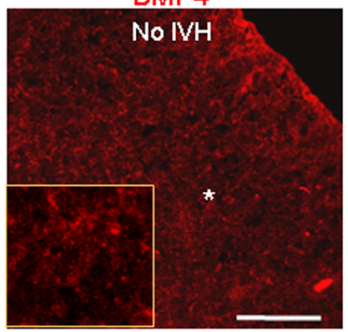

BMP4 Tui-1

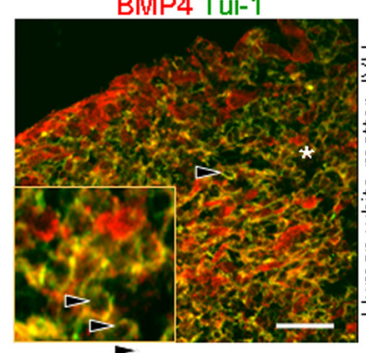

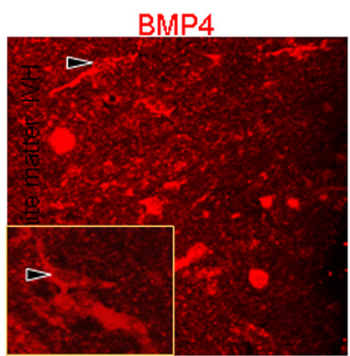

BMP4 NG2

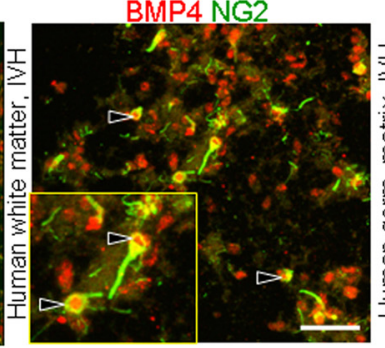

BMP4 Oliq2

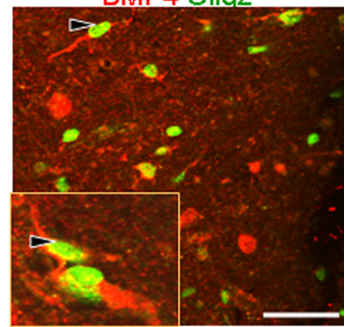

BMP4 GFAP

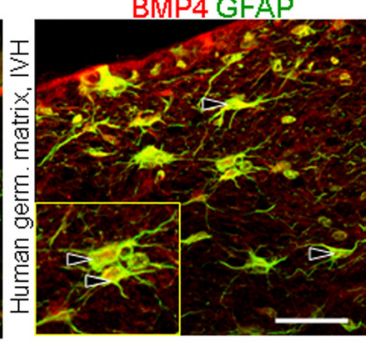

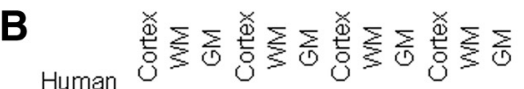
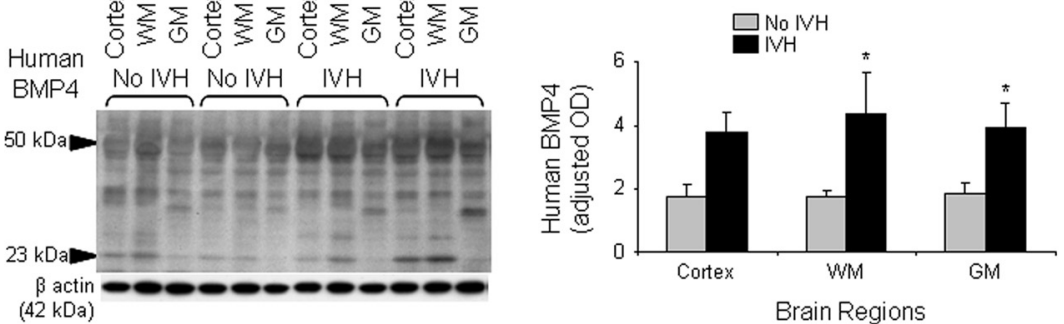

Figure 7. IVH induces elevation of BMP4 in premature infants. $A$, Representative immunofluorescence of cryosections from premature infants (23 week gestation) with and without IVH labeled with BMP4 antibody (top). Insets show high-magnification views from the region in the image indicated by asterisk. Immunoreactivity to BMP4 is more abundant in IVH subjects than controls without IVH. Cryosections from 23 week human premature infant were labeled with a combination of BMP4 with Olig2-, Tuj1-, NG2-, or GFAP-specific antibodies. Note BMP4 immunoreactivity in Tuj1-positive neuronal precursors (arrowheads) of the VZ and SVZ as well as Olig2-positive (arrowheads) and NG2-positive 0L. BMP4 was also expressed in GFAP ( + ) astrocytes in the white matter and cortex. Scale bar, $50 \mu \mathrm{m} . \boldsymbol{B}$, Representative Western blot analyses for BMP4 in postmortem brain samples from premature infants. Values are normalized to $\beta$-actin levels. Error bars are mean \pm SEM ( $n=6$ each time point). BMP4 [both precursor $(50 \mathrm{kDa}$ ) and mature $(23 \mathrm{kDa})$ form] expression was higher in the germinal matrix (GM) (VZ and SVZ) and white matter (WM) of premature infants with IVH compared with infants without IVH. ${ }^{*} p<0.05$ for the comparison between subjects with and without IVH. Western blot analyses revealed that BMP4 protein levels were significantly higher in the VZ and SVZ (germinal matrix, $p=0.048$ ) as well as in the white matter $(p<0.014$ ) of the infants with IVH relative to infants without IVH, but not in the cerebral cortex ( $p=0.07)$ (Fig. 7B).

with IVH compared with vehicle-treated and untreated controls with IVH $(p<0.001$ and 0.045$)$ (Fig. $8 B)$. Similarly, the volume fraction of MBP in the corona radiata was higher in the noggintreated pups compared with vehicle-treated controls $(p<$ 0.005 ), but not with untreated IVH controls ( $p=0.06$ ). Accordingly, Western blot analyses showed that noggin treatment of pups with IVH significantly enhanced the expression of MBP compared with vehicle-treated and untreated pups with IVH $(p<0.05$ each) (Fig. 8C).

To further confirm the effect of noggin treatment on myelination, we compared the expression of MAG in noggin-treated, vehicle-treated, and untreated pups with IVH as well as pups without IVH. MAG levels were significantly elevated in noggintreated rabbit pups with IVH compared with vehicle-treated and untreated controls with IVH ( $p=0.001$ both) (Fig. $8 D$ ). Collectively, noggin treatment significantly enhanced MBP and MAG expression in pups with IVH.

\section{Noggin treatment reduces gliosis}

We next compared gliosis among four sets of P14 rabbit pups in a similar fashion as for myelin. GFAP-labeled brain sections were evaluated by unbiased stereology and quantification of GFAP in homogenates from brain slice (midseptal nucleus level) was performed by Western blot analyses. For stereological assessment of gliosis, we performed astrocyte count and measured total volume fraction of astrocytes and glial fibers in GFAP-labeled brain sections. We found that there were fewer astrocytes in the corpus callosum and corona radiata of noggin-treated pups with IVH compared with vehicle-treated controls with IVH $(p<0.028$ for corpus callosum and 0.017 for corona radiate) (Fig. 9A). Accordingly, the total volume faction (load) of astrocyte cell bodies and their fibers were significantly reduced in the corpus callosum $(p<0.001$ and 0.008 for noggin- vs vehicle-treated or untreated IVH controls) and corona radiata ( $p<0.001$ both for noggin- vs vehicle-treated or untreated IVH controls) of noggin-treated pups with IVH compared with vehicle-treated and untreated controls with IVH.

Consistent with stereological evaluation, Western blot analyses revealed that GFAP levels were significantly reduced in noggin-treated pups compared with vehicle-treated and untreated controls ( $p<0.001$ both) (Fig. 9B). Furthermore, GFAP levels were comparable between noggin-treated pups with IVH and controls without IVH. To determine the effect of noggin on astrocyte proliferation, we double labeled the brain sections from pups without IVH, with IVH, and with IVH treated with noggin using Ki67 and GFAP-specific antibodies. We found that noggin treatment did not affect astrocyte proliferation (data not shown). 
A

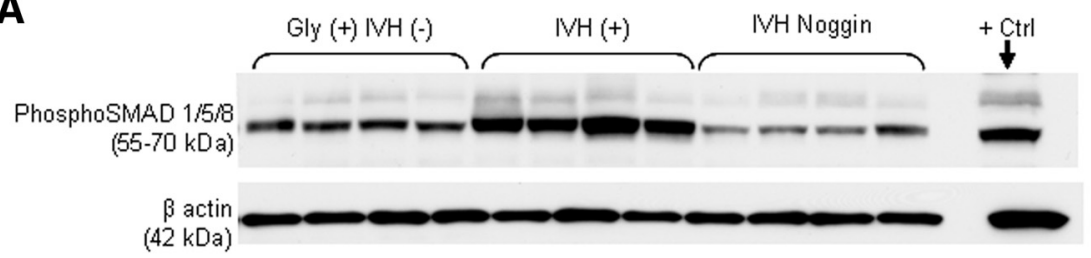

B
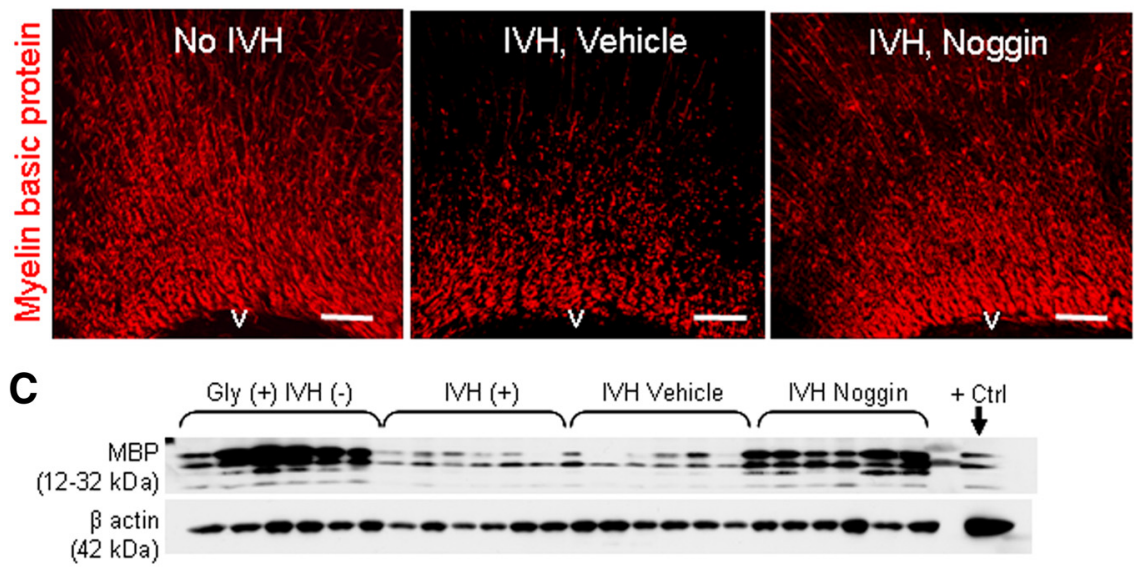

D

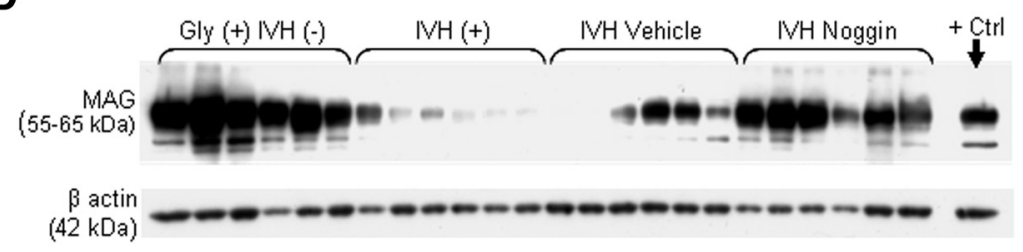

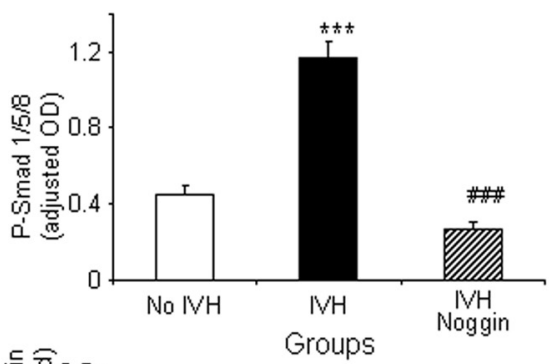
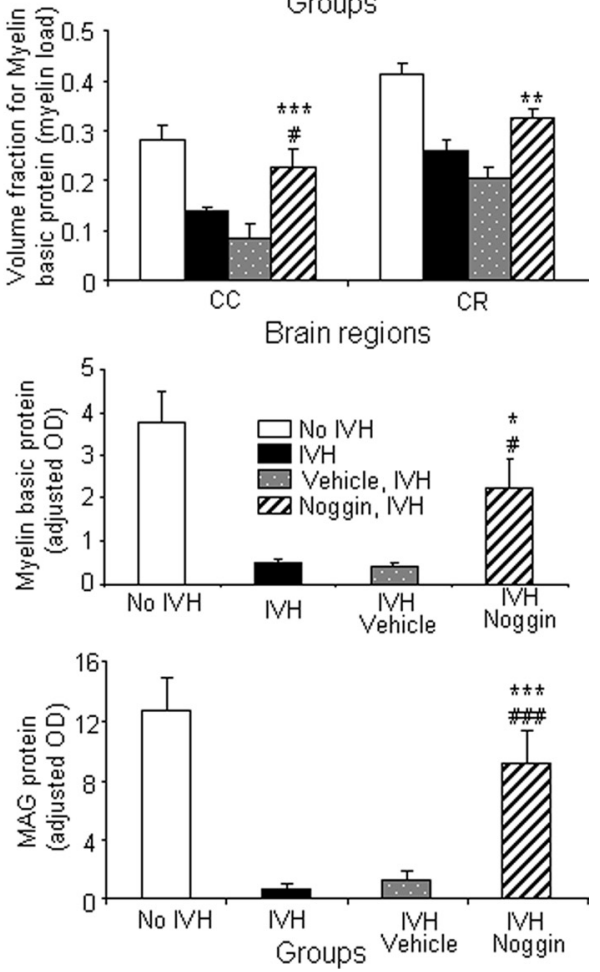

Figure 8. Noggin treatment restores phospho-Smad1/5/8 and enhances myelination. $\boldsymbol{A}$, Phospho-Smad1/5/8 elevated in IVH and noggin treatment reduced its expression. Representative Western blot analyses for phospho-Smad1/5/8 in the forebrain of noggin- and vehicle-treated pups with IVH as well as pups without IVH. Each lane represents lysate from whole coronal slice taken at the level of midseptal nucleus of one brain. Positive control (Ctrl) was lysate made from rat glial C6 cells treated with BMP4. The data are mean \pm SEM. Phospho-Smad1/5/8 expression was significantly higher in rabbit pups with IVH compared with pups without IVH; and noggin treatment reduced phospho-Smad1/5/8 levels in pups with IVH. ${ }^{* * *} p<0.001$ for the comparison between pups with and without IVH. ${ }^{\# \# \#}<0.001$ for the comparison between noggin-treated and vehicle-treated pups with IVH. Gly, Glycerol. $\boldsymbol{B}$, Representative immunofluorescence of cryosections from 14-d-old pups labeled with MBP-specific antibody. Note reduced immunoreactivity to MBP in periventricular zone (corona radiata) of pups with IVH and restoration of myelin in noggin-treated pups. Error bars are mean \pm SEM ( $n=5$ - 6 each time point). Volume fraction of MBP was higher in corpus callosum (CC) of noggin-treated pups compared with vehicle-treated and untreated control pups with IVH. Volume fraction of MBP in the corona radiata was also more abundant in the noggin-treated pups than in vehicle-treated controls, but not in untreated IVH controls. Scale bar, $100 \mu \mathrm{m}$. $\mathrm{V}$, ventricle. C, Representative Western blot analyses for MBP in the forebrain of premature rabbit pups in four sets of pups as indicated. A healthy mouse brain was used as positive control. Each lane represents lysate from whole coronal slice taken at the level of midseptal nucleus of one brain. Error bars are mean \pm SEM ( $n=5-6$ each group). MBP expression was higher in noggin-treated pups compared with vehicle-treated pups. $\boldsymbol{D}$, Representative Western blot analyses for MAG in the forebrain of premature rabbit pups in four sets of pups as indicated. Rat brain was used as positive control. The data in the bar diagram are mean \pm SEM ( $n=6$ each group). MAG expression was higher in noggin-treated pups compared with vehicle-treated and untreated pups with hemorrhage. ${ }^{* * *} p<0.001,{ }^{* *} p<0.01$, and ${ }^{*} p<0.05$ for the comparison between noggin-treated pups and vehicle-treated pups. ${ }^{\# \# \#} p<0.001$ and ${ }^{\#} p<0.05$ for the comparison between noggin-treated pups and untreated pups.

Together, noggin treatment not only enhanced myelination, but also reduced gliosis in rabbit pups with IVH.

\section{Noggin restores Olig2 and Id4 levels, and enhances maturation of $\mathrm{OL}$}

As BMP signaling suppresses OL maturation by inducing $\mathrm{Id} 2$ and Id4, and suppressing Olig1 as well as Olig2 transcription factors (Samanta and Kessler, 2004), we hypothesized that the BMP antagonist, noggin, would restore levels of Olig1 and Olig2 transcription factor. We found that Olig2 mRNA expression was significantly higher in noggin-treated pups compared with vehicle controls at days 3 and 7 ( $p=0.047$ and 0.002 ), but not at day $14(p=0.7)$ (Fig. 10A). Accordingly Id4 mRNA abundance was substantially lower in all noggin-treated pups relative to vehicle controls ( $p=0.011$ ) when all subjects at three time points- days 3,7 and $14-$ were combined. However, difference between the two groups at any particular time point (days 3,7 , or 14) was not significant $(p=0.19,0.06,0.47)$. Accordingly, Sox10 levels trended higher at days 3 and 7 in treated pups relative to vehicle controls, however the statistical comparison was not significant. Olig1 and Id2 gene expression were comparable between the two groups. As IVH-induced Id 4 and to a lesser extent Id 2 (only at day 1 ), as well as suppressed Sox10, Olig2, but not Olig1, noggin treatment seemingly offsets the levels of only those transcription factors that are altered in pups with IVH. Collectively, noggin treatment restored Olig2 and Id4 transcription factors in pups with IVH.

We next compared the density of pre-OL and immature OLs between noggin- and vehicle-treated pups with IVH. Double immu- 

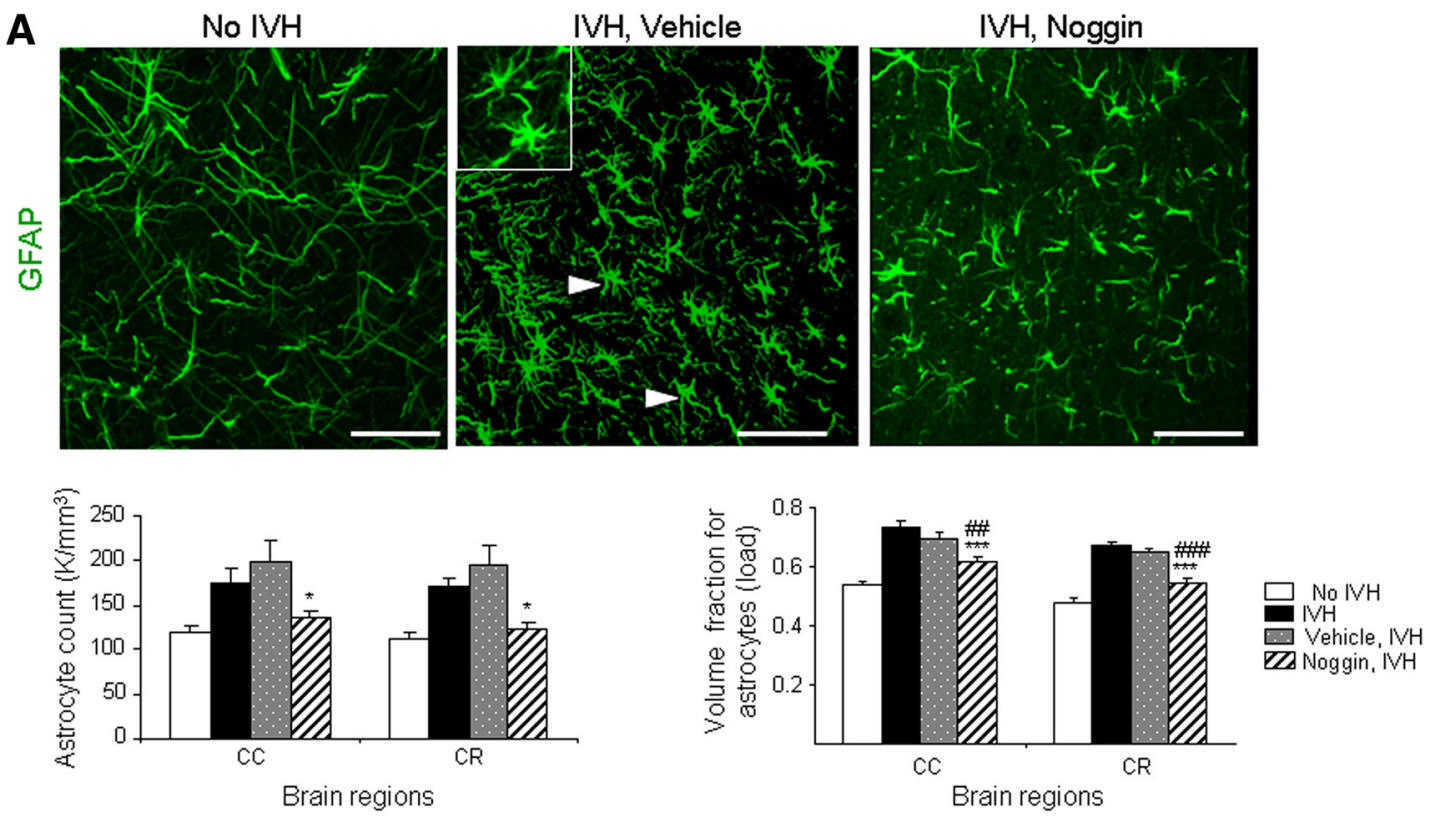

B
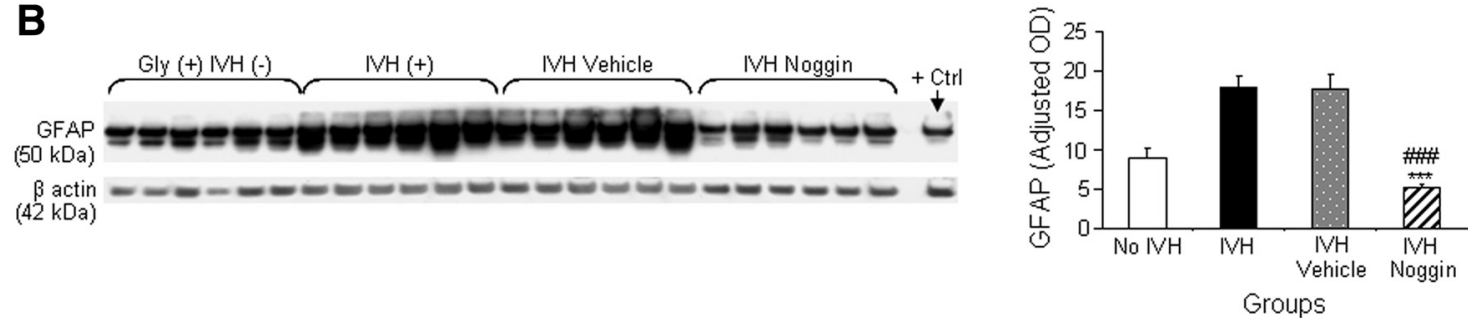

Figure 9. Noggin treatment reduces gliosis. $\boldsymbol{A}$, Representative labeling of cryosections from 14-d-old rabbit pups labeled with GFAP antibody. Note abundant hypertrophic astrocytes-with large cell body and numerous processes making a dense network -in the periventricular zone of pups with IVH (arrowheads) and reduction in number of these astrocytes in noggin-treated pups. Inset shows hypertrophic astrocytes. The bars are mean \pm SEM ( $n=5-6$ each group). Astrocyte count in the corpus callosum (CC) and corona radiata (CR) was less in noggin-treated pups with IVH compared with vehicle-treated controls with IVH. The total volume fraction of astrocyte cell body and their fibers was less abundant in corona radiata and corpus callosum of noggin-treated pups with IVH compared with vehicle-treated and untreated control pups with IVH. B, Representative Western blot analyses for GFAP in the forebrain of premature rabbit pups in four groups of pups as indicated. The data are mean \pm SEM ( $n=6$ each group). A healthy rat brain was used as positive control. Each lane represents lysate from whole coronal slice taken at the level of midseptal nucleus of one brain. GFAP expression was lower in noggin-treated pups compared with vehicle-treated and untreated pups. ${ }^{* *} p<0.001,{ }^{* *} p<0.01$, and ${ }^{*} p<0.05$ for the comparison between noggin-treated pups and vehicle-treated pups. ${ }^{\# \#} p<0.001$ for the comparison between noggin-treated pups and untreated pups. Scale bar, $50 \mu \mathrm{m}, \mathrm{K}=10^{3}$.

nostaining with $\mathrm{O} 4$ and $\mathrm{O} 1$ antibodies showed that pre-OLs at day 3 were fewer in the corona radiata of noggin-treated than vehicletreated pups $(p<0.001)$, but not in the corpus callosum and internal capsule ( $p=0.143,0.562$ ) (Fig. $10 B)$. At day 7 , density of pre-OL was comparable between noggin- and vehicle-treated pups with IVH in all the three white matter regions. Importantly, immature OLs were more abundant in noggin-treated pups compared with vehicle-treated pups at both day $3(p<0.001,0.001$, and 0.032$)$ and day $7(p<0.001$, 0.001 , and 0.009 ) (Fig. 10B) in all the three brain regions. This suggested that noggin treatment augmented maturation of pre-OL into immature $\mathrm{OL}$, leading to a reduction in the density of pre-OL.

We next assessed proliferation of Olig2 $(+)$ cells in noggintreated IVH pups compared with vehicle-treated controls by double labeling sections with Olig2 and Ki67 antibody. We found that noggin treatment did not affect proliferation of Olig2 $(+)$ cells in the SVZ of IVH pups compared with vehicle controls at day 3 (142.8 \pm 26.8 vs $132.5 \pm 10.0, p=0.7$ ), which is consistent with the previous report (Cate et al., 2010). To determine an effect of noggin treatment on OL population in healthy animals, we alternately treated healthy E29 rabbit pups (no IVH) with human recombinant noggin or vehicle for $3 \mathrm{~d}$. The density of Olig2-positive cells was comparable between noggin- and vehicle-treated pups without IVH at day 3 , suggesting that noggin treatment in healthy animals may not affect OL population (Fig. 10C).
As posthemorrhagic hydrocephalus is an important outcome of IVH in our animal model, just like premature infants, we asked whether placement of ICV cannula affected ventriculomegaly in the ICV noggin- and ICV vehicle-treated rabbit pups compared with untreated controls. Based on our previous study (Chua et al., 2009), ventriculomegaly was defined as a ventricular area that measures $>3$ $\mathrm{SD}$ above the mean for age in non-IVH pups. The incidence of ventriculomegaly was $7 \%$ in noggin-treated, $14 \%$ in vehicle-treated and $40 \%$ in untreated rabbit pups. The reduction in ventriculomegaly in noggin- and vehicle-treated in contrast to the untreated pups suggested that indwelling ICV cannula decompressed the lateral ventricle. To confirm the CSF leakage around the ICV cannula, we infused two rabbit pups with Evan's blue using the osmotic pumps. Blue staining on the surface of the cerebral cortex and periosteal surface around the cannula suggested decompression of the ventricle by the ICV cannula in vehicle- and noggin-treated pups. Together, noggin treatment did not affect cerebral ventricle size, but the placement of the ICV cannula reduced the ventricle size.

\section{Noggin treatment enhances density of myelinated axons in pups with IVH on ultrastructural study}

To determine morphological recovery in myelination upon noggin treatment in pups with IVH, we evaluated myelin at ultrastructural level in 3 sets of pups: a) glycerol-treated pups without 
$\mathrm{IVH}, \mathrm{b})$ untreated pups with $\mathrm{IVH}$, and c) noggin-treated pups with IVH (Fig. 11). An evaluation of white matter of pups with IVH revealed that the myelinated and unmyelinated fibers, in general, were well organized and preserved, similar to controls without IVH. However, a few axons in pups with IVH displayed features of axonal degeneration, including intraaxonal vacuoles and autophagosomes, which were not seen in pups without IVH. We did not identify Wallerian-like axonal degeneration. In addition, remyelinating axons and very small axons representing regenerating sprouts were not seen. We next compared the density of myelinated axons in the three groups of pups. We found that myelinated axons were fewer in pups with IVH compared with controls without IVH $(p=0.024)$. In addition, noggin treatment significantly increased the number of myelinated axons in pups with IVH $(p<0.05)$. However, g-ratio was comparable in the three groups of pups $(0.77 \pm 0.01$ vs $0.76 \pm 0.001$ vs $0.76 \pm 0.014$, in pups without IVH, with IVH and noggin-treated respectively). Usually, axons of larger diameter have larger g-ratio, that is, they have relatively a thinner myelin sheath. Together, the density of myelinated axons is reduced in pups with IVH, and noggin treatment restored their density.

\section{Discussion}

IVH is a major neurological disorder of premature infants that results in cerebral palsy and cognitive deficits. As the prematurity rate is increasing and since the survival of these infants has markedly improved, neurologic sequelae following IVH is a major public health concern (Arias et al., 2003; Jain et al., 2009). In this study, we explored the mechanistic basis of white matter injury in IVH and exploited a novel strategy to protect the white matter. Specifically, we found that the induction of IVH in premature rabbit pups resulted in increased apoptosis, reduced proliferation and maturational failure of OL-lineage cells in the periventricular white matter and that inhibition of BMP by noggin treatment led to (1) restoration of Olig2 and Id4 transcription factors, (2) maturation of preOL, (3) improved myelination, (4) reduced astrocytosis, and (5) neurological recovery.

In the present study, IVH resulted in apoptosis, reduced proliferation and maturational failure of OL progenitors. Apoptosis was associated with significant elevation in capsase-3 at day 3 and 7, but not at day 14 . This suggested IVH-associated early apoptosis to be caspase-dependent and late apoptosis to be caspase-independent. In our IVH model, we have previously shown an elevation of caspase- $3 / 7,-8$, and -9 activities at 12,48 , and $72 \mathrm{~h}$ of age, indicating activation of both intrinsic and extrinsic apoptotic pathways in IVH (Vinukonda et al., 2010). Caspase-independent forms of apoptosis at day 14 in our model might be ascribed to mitochondrial release of apoptosisinducing-factor and perhaps of other proteins, as reported in animal models of focal cerebral ischemic damage (Culmsee et al.,
2005; Krantic et al., 2007). Our finding of reduced neural cell proliferation in pups with IVH is in agreement with the report in blood infusion model of IVH in rats (Xue et al., 2003). Importantly, we noted an elevation in the density of pre-OLs $(\sim 10-$ $20 \%$ ) in pups with IVH, despite their apoptosis and reduction in proliferation. We attribute this primarily to arrested maturation of pre-OL into immature OL resulting in escalation in pre-OL and reduction in immature OL density. The suppression in OL proliferation was transient (at day 3, but not at day 7). Thus, maturational arrest of pre-OL along with their degeneration appears to be the major event undergoing in the white matter of pups with IVH. Similar observation of the maturational arrest of pre-OL has been made in a recent study in a rat model of hypoxiaischemia (Segovia et al., 2008) and indicated in a study on autopsy materials of premature infants with periventricular leukomalacia (Billiards et al., 2008). However, hypoxia-ischemia induces rapid proliferation of OL progenitors (Segovia et al., 2008), in contrast to our IVH model where reduction in mitosis was noted. The reduction in maturation of OL is unlikely to be related to a loss of axons because axons are relatively preserved in our model of IVH. However, we did not exclude the possibility of modification of proteins in intact appearing axons, which might affect the differentiation of pre-OLs and axonal myelination (Haynes et al., 2005). Together, hypomyelination in premature infants with IVH could be the result of apoptosis and arrested maturation of OL lineage. 


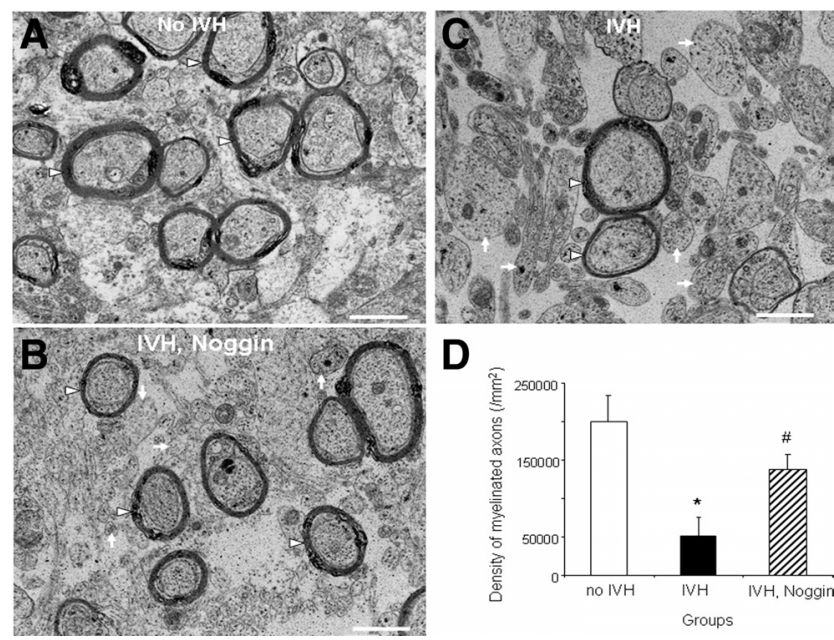

Figure 11. Noggin treatment enhances density of myelinated axons in pups with IVH at ultrastructural level. $\boldsymbol{A}-\boldsymbol{C}$, Electron micrographs showing axons in the corona radiata of rabbit pups without IVH $(\boldsymbol{A})$, untreated pup with IVH $(\boldsymbol{B})$, and noggin-treated pup with IVH (C). Note myelinated (arrowheads) and unmyelinated (arrows) axons in the images. D, The bar chart shows mean and SEM. The myelinated axons (arrowheads) were fewer in pups with IVH compared with controls without IVH; and noggin treatment increased the density of myelinated axons in pups with IVH. Scale bar, $1 \mu \mathrm{m}$. ${ }^{*} p<0.05$ for the comparison between pups with and without IVH. ${ }^{\#} p<0.05$ for the comparison between noggin-treated and untreated pups with IVH.

A number of studies have revealed that BMP overexpression inhibits differentiation of OL and diverts OL progenitors to astrocyte lineage, thus promoting astrocytosis (Gomes et al., 2003; Liu and Niswander, 2005; Hampton et al., 2007). Consistent with these findings, we noted an elevation in BMP4 protein associated with maturational failure of OL progenitors and gliosis in our rabbit model of IVH. Accordingly, an elevation in levels of phospho-Smad1/5/8 in rabbit pups with IVH showed more enhanced BMP signaling in pups with IVH compared with pups without IVH. Importantly, premature human infants with IVH also exhibited high BMP4 levels compared with infants without IVH. To our knowledge, this is the first report demonstrating elevation of BMP4 in an animal model of neonatal brain injury and premature human infants with IVH. Although human data from autopsy materials are invaluable, they have inherent limitations because of the associated cofounding variables, including mechanical ventilation, exposure to a number of prenatal and postnatal medications, and others. High BMP levels in the brain region around the ventricle are likely to act locally in the SVZ and in the subjacent white matter since it binds to the extracellular matrix, limiting its diffusion through tissues (Hall and Miller, 2004). As OL progenitors in the white matter and cortex originate from the VZ and SVZ of human fetuses (Rakic and Zecevic, 2003), high BMP in the periventricular white matter could arrest maturation of OL in the pre-OL stage and augment gliosis in this brain region. Of note, white matter disease in premature infants and also in our rabbit model is seen predominantly in the periventricular white matter (Okoshi et al., 2001). Hence, arrested maturation of OL after IVH appears to be BMP dependent. Even though BMP4 enhances apoptosis in certain context, apoptosis after IVH appears to be BMP independent.

The most important and novel observation of this study was the neurological recovery in premature rabbit pups with IVH by the use of BMP inhibitor, noggin. In fact, this is the first use of recombinant human noggin as a neuroprotectant in an animal model of neonatal brain injury. BMP2 and BMP4 are the major types of BMP expressed in the forebrain; and noggin is an extracellular BMP antagonist that binds with BMP2/4, thereby interfering with its binding to receptors. BMP4 treatment on cerebral OL precursor cells reduces MBP and proteolipid protein expression via activation of Id-2 and Id-4 and inhibition of Olig1 and Olig2 genes (Yanagisawa et al., 2001; Cheng et al., 2007); and conversely, overexpression of BMP antagonist - noggin — in genetically engineered mice reduces ischemic brain injury by increasing the density of OL progenitors (Samanta et al., 2010). Furthermore, chordin, a BMP antagonist, promotes oligodendrogenesis in the SVZ cells in both in vivo and in vitro experiments (Jablonska et al., 2010). Consistent with these published data, we found that noggin treatment enhanced the maturation of pre-OLs into immature OLs and thereby augmented myelination. In addition, Olig2 levels were significantly elevated and Id4 expression was more reduced in noggin-treated than in vehicletreated control pups in our animal model. Together, the data suggest that noggin treatment promoted maturation of pre-OLs into myelinating OLs by upregulating Olig2 and downregulating Id4 transcription factor, thereby promoting myelination and neurological recovery.

Another key finding that emerged from our study was that noggin treatment does not only promote myelination, but significantly reduces gliosis. Consistent with our finding, it has been shown in adult rat spinal cord that a local increase in BMPs at the site of demyelination results in gliosis, glial scar formation and elevation in the expression of chondroitin sulfate proteoglycans (CSPG) (Fuller et al., 2007). Heightened expression of CSPGs, including versican, neurocan and aggrecan, inhibit proliferation of OLs and myelination (Asher et al., 2002); and conversely, degradation of CSPGs by either chondroitinase or protease facilitates migration of OLs (Ikegami et al., 2005), loosens the physical barriers in extracellular matrix (Silver and Miller, 2004), enhances formation of OLs process outgrowths, and improves interaction between growth factors and OLs (Oh et al., 1999). Hence, neurological recovery with noggin treatment might be partly attributed to reduction in gliosis and resultant attenuation of CSPG levels in the extracellular matrix of the white matter.

Moderate to severe IVH in premature infants results in cerebral palsy and cognitive deficits in a large number of the survivors. Even though infrequent, active withdrawal of life support from premature infants with severe IVH does happen, based on the quality of life concerns (Sawyer, 2008). At this time, no treatment is available for this disorder. Thus, our study showing clinical recovery in treated pups compared with untreated controls offers a major strategy that can potentially be translated in patient treatment. A major hurdle to our approach is the requirement of intracerebral rather than systemic administration of the medication to downregulate BMP4 in the brain. However, ventricular drainage using frontal and occipital catheters, irrigation with artificial CSF, and fibrinolytic therapy have been tried in a randomized clinical trial in premature infants without significant success (Whitelaw et al., 2007). Hence, intracerebral administration of BMP inhibitors in the cerebral ventricle of premature infants, even though invasive and involving risk, is plausible. Systemic use of small molecule BMP inhibitors that penetrates the blood brain barrier has not undergone clinical trial for any disease so far (Hong and $\mathrm{Yu}, 2009$ ). If intracerebral treatment of noggin proves to be safe and effective in preventing neurologic sequelae in premature infants with IVH, then its use can potentially have a major impact on the neurological integrity and developmental outcome of premature infants. 


\section{References}

Arias E, MacDorman MF, Strobino DM, Guyer B (2003) Annual summary of vital statistics-2002. Pediatrics 112:1215-1230.

Armstrong DL, Sauls CD, Goddard-Finegold J (1987) Neuropathologic findings in short-term survivors of intraventricular hemorrhage. Am J Dis Child 141:617-621.

Asher RA, Morgenstern DA, Shearer MC, Adcock KH, Pesheva P, Fawcett JW (2002) Versican is upregulated in CNS injury and is a product of oligodendrocyte lineage cells. J Neurosci 22:2225-2236.

Back SA, Luo NL, Borenstein NS, Levine JM, Volpe JJ, Kinney HC (2001) Late oligodendrocyte progenitors coincide with the developmental window of vulnerability for human perinatal white matter injury. J Neurosci 21:1302-1312.

Back SA, Luo NL, Mallinson RA, O’Malley JP, Wallen LD, Frei B, Morrow JD, Petito CK, Roberts CT Jr, Murdoch GH, Montine TJ (2005) Selective vulnerability of preterm white matter to oxidative damage defined by F2-isoprostanes. Ann Neurol 58:108-120.

Ballabh P, Xu H, Hu F, Braun A, Smith K, Rivera A, Lou N, Ungvari Z, Goldman SA, Csiszar A, Nedergaard M (2007) Angiogenic inhibition reduces germinal matrix hemorrhage. Nat Med 13:477-485.

Bassan H, Limperopoulos C, Visconti K, Mayer DL, Feldman HA, Avery L, Benson CB, Stewart J, Ringer SA, Soul JS, Volpe JJ, du Plessis AJ (2007) Neurodevelopmental outcome in survivors of periventricular hemorrhagic infarction. Pediatrics 120:785-792.

Billiards SS, Haynes RL, Folkerth RD, Borenstein NS, Trachtenberg FL, Rowitch DH, Ligon KL, Volpe JJ, Kinney HC (2008) Myelin abnormalities without oligodendrocyte loss in periventricular leukomalacia. Brain Pathol 18:153-163.

Buffo A, Vosko MR, Ertürk D, Hamann GF, Jucker M, Rowitch D, Götz M (2005) Expression pattern of the transcription factor Olig2 in response to brain injuries: implications for neuronal repair. Proc Natl Acad Sci U S A 102:18183-18188.

Bystron I, Blakemore C, Rakic P (2008) Development of the human cerebral cortex: Boulder Committee revisited. Nat Rev Neurosci 9:110-122.

Cate HS, Sabo JK, Merlo D, Kemper D, Aumann TD, Robinson J, Merson TD, Emery B, Perreau VM, Kilpatrick TJ (2010) Modulation of bone morphogenic protein signalling alters numbers of astrocytes and oligodendroglia in the subventricular zone during cuprizone-induced demyelination. J Neurochem 115:11-22.

Cheng X, Wang Y, He Q, Qiu M, Whittemore SR, Cao Q (2007) Bone morphogenetic protein signaling and olig1/2 interact to regulate the differentiation and maturation of adult oligodendrocyte precursor cells. Stem Cells 25:3204-3214.

Chua CO, Chahboune H, Braun A, Dummula K, Chua CE, Yu J, Ungvari Z, Sherbany AA, Hyder F, Ballabh P (2009) Consequences of intraventricular hemorrhage in a rabbit pup model. Stroke 40:3369-3377.

Culmsee C, Zhu C, Landshamer S, Becattini B, Wagner E, Pellechia M, Blomgren K, Plesnila N (2005) Apoptosis-inducing factor triggered by poly(ADP-ribose) polymerase and Bid mediates neuronal cell death after oxygen-glucose deprivation and focal cerebral ischemia. J Neurosci 25:10262-10272.

Fuller ML, DeChant AK, Rothstein B, Caprariello A, Wang R, Hall AK, Miller RH (2007) Bone morphogenetic proteins promote gliosis in demyelinating spinal cord lesions. Ann Neurol 62:288-300.

Georgiadis P, Xu H, Chua C, Hu F, Collins L, Huynh C, Lagamma EF, Ballabh P (2008) Characterization of acute brain injuries and neurobehavioral profiles in a rabbit model of germinal matrix hemorrhage. Stroke 39:3378-3388.

Gomes WA, Mehler MF, Kessler JA (2003) Transgenic overexpression of BMP4 increases astroglial and decreases oligodendroglial lineage commitment. Dev Biol 255:164-177.

Hall AK, Miller RH (2004) Emerging roles for bone morphogenetic proteins in central nervous system glial biology. J Neurosci Res 76:1-8.

Hampton DW, Asher RA, Kondo T, Steeves JD, Ramer MS, Fawcett JW (2007) A potential role for bone morphogenetic protein signalling in glial cell fate determination following adult central nervous system injury in vivo. Eur J Neurosci 26:3024-3035.

Haynes RL, Borenstein NS, Desilva TM, Folkerth RD, Liu LG, Volpe JJ, Kinney HC (2005) Axonal development in the cerebral white matter of the human fetus and infant. J Comp Neurol 484:156-167.

Heuchan AM, Evans N, Henderson Smart DJ, Simpson JM (2002) Perinatal risk factors for major IVH in the Australian and New Zealand Neonatal Network, 1995-97. Arch Dis Child Fetal Neonatal Ed 86:F86-F90.

Hong CC, Yu PB (2009) Applications of small molecule BMP inhibitors in physiology and disease. Cytokine Growth Factor Rev 20:409-418.

Ikegami T, Nakamura M, Yamane J, Katoh H, Okada S, Iwanami A, Watanabe K, Ishii K, Kato F, Fujita H, Takahashi T, Okano HJ, Toyama Y, Okano H (2005) Chondroitinase ABC combined with neural stem/progenitor cell transplantation enhances graft cell migration and outgrowth of growthassociated protein-43-positive fibers after rat spinal cord injury. Eur J Neurosci 22:3036-3046.

Jablonska B, Aguirre A, Raymond M, Szabo G, Kitabatake Y, Sailor KA, Ming GL, Song H, Gallo V (2010) Chordin-induced lineage plasticity of adult SVZ neuroblasts after demyelination. Nat Neurosci 13:541-550.

Jain NJ, Kruse LK, Demissie K, Khandelwal M (2009) Impact of mode of delivery on neonatal complications: trends between 1997 and 2005. J Matern Fetal Neonatal Med 22:491-500.

Krantic S, Mechawar N, Reix S, Quirion R (2007) Apoptosis-inducing factor: a matter of neuron life and death. Prog Neurobiol 81:179-196.

Liu A, Niswander LA (2005) Bone morphogenetic protein signalling and vertebrate nervous system development. Nat Rev Neurosci 6:945-954.

Lu QR, Sun T, Zhu Z, Ma N, Garcia M, Stiles CD, Rowitch DH (2002) Common developmental requirement for Olig function indicates a motor neuron/oligodendrocyte connection. Cell 109:75-86.

Mouton PR, Chachich ME, Quigley C, Spangler E, Ingram DK (2009) Caloric restriction attenuates amyloid deposition in middle-aged dtg APP/ PS1 mice. Neurosci Lett 464:184-187.

Nicolay DJ, Doucette JR, Nazarali AJ (2007) Transcriptional control of oligodendrogenesis. Glia 55:1287-1299.

Oh LY, Larsen PH, Krekoski CA, Edwards DR, Donovan F, Werb Z, Yong VW (1999) Matrix metalloproteinase-9/gelatinase B is required for process outgrowth by oligodendrocytes. J Neurosci 19:8464-8475.

Okoshi Y, Itoh M, Takashima S (2001) Characteristic neuropathology and plasticity in periventricular leukomalacia. Pediatr Neurol 25:221-226.

Pfeiffer SE, Warrington AE, Bansal R (1993) The oligodendrocyte and its many cellular processes. Trends Cell Biol 3:191-197.

Rakic S, Zecevic N (2003) Early oligodendrocyte progenitor cells in the human fetal telencephalon. Glia 41:117-127.

Samanta J, Kessler JA (2004) Interactions between ID and OLIG proteins mediate the inhibitory effects of BMP4 on oligodendroglial differentiation. Development 131:4131-4142.

Samanta J, Alden T, Gobeske K, Kan L, Kessler JA (2010) Noggin protects against ischemic brain injury in rodents. Stroke 41:357-362.

Sawyer T (2008) Withdrawing support for withdrawing support from premature infants with severe intracranial hemorrhage. Pediatrics 121:1071-1072.

See J, Mamontov P, Ahn K, Wine-Lee L, Crenshaw EB 3rd, Grinspan JB (2007) BMP signaling mutant mice exhibit glial cell maturation defects. Mol Cell Neurosci 35:171-182.

Segovia KN, McClure M, Moravec M, Luo NL, Wan Y, Gong X, Riddle A, Craig A, Struve J, Sherman LS, Back SA (2008) Arrested oligodendrocyte lineage maturation in chronic perinatal white matter injury. Ann Neurol 63:520-530.

Silver J, Miller JH (2004) Regeneration beyond the glial scar. Nat Rev Neurosci 5:146-156.

Vinukonda G, Csiszar A, Hu F, Dummula K, Pandey NK, Zia MT, Ferreri NR, Ungvari Z, LaGamma EF, Ballabh P (2010) Neuroprotection in a rabbit model of intraventricular haemorrhage by cyclooxygenase-2, prostanoid receptor-1 or tumour necrosis factor-alpha inhibition. Brain 133:2264-2280.

Whitelaw A, Evans D, Carter M, Thoresen M, Wroblewska J, Mandera M, Swietlinski J, Simpson J, Hajivassiliou C, Hunt LP, Pople I (2007) Randomized clinical trial of prevention of hydrocephalus after intraventricular hemorrhage in preterm infants: brain-washing versus tapping fluid. Pediatrics 119:e1071-e1078.

Xin M, Yue T, Ma Z, Wu FF, Gow A, Lu QR (2005) Myelinogenesis and axonal recognition by oligodendrocytes in brain are uncoupled in Olig1null mice. J Neurosci 25:1354-1365.

Xue M, Balasubramaniam J, Buist RJ, Peeling J, Del Bigio MR (2003) Periventricular/intraventricular hemorrhage in neonatal mouse cerebrum. J Neuropathol Exp Neurol 62:1154-1165.

Yanagisawa M, Takizawa T, Ochiai W, Uemura A, Nakashima K, Taga T (2001) Fate alteration of neuroepithelial cells from neurogenesis to astrocytogenesis by bone morphogenetic proteins. Neurosci Res 41:391-396. 